\title{
Developments of New Sheet Metal Forming Technology and Theory in China
}

\author{
Shi-Hong Zhang ${ }^{1} \cdot$ Shuai-Feng Chen ${ }^{1} \cdot{\text { Yan } \mathrm{Ma}^{1} \cdot \text { Hong-Wu Song }}^{1} \cdot$ Ming Cheng $^{1}$
}

Received: 4 July 2015/Revised: 7 November 2015/Published online: 8 December 2015

(C) The Chinese Society for Metals and Springer-Verlag Berlin Heidelberg 2015

\begin{abstract}
Developments of new sheet metal forming technology and theory in China are reviewed in detail in this paper. Advances of crystal plasticity on the deformation mechanism of $\mathrm{Mg}$ alloy are firstly described, especially its applications on the prediction of sheet forming process. Then, a new macroscopic constitutive model is introduced, which possesses an enhanced description capacity of tension/compression anisotropy and anisotropic hardening. In order to take into account the twinning process of hexagonal close-packed material, a modified hierarchical multi-scale model is also established with adequate accuracy in a shorter computational time. The advanced forming limit of sheet metal, mainly about aluminum alloy, is also investigated. Besides the above theory developments, some new sheet metal forming technologies are reviewed simultaneously. The warm forming technology of $\mathrm{Mg}$ alloy is discussed. New processes to form sheet parts and to bend tubes are proposed by using hard granules. On the other hand, a new kind of ultra-high-strength steel based on typical $22 \mathrm{MnB} 5$ by introducing more residual austenite and $\mathrm{Cu}$-rich phase to increase the elongation and strength and its novel forming method that integrates hot stamping and quenching participation are proposed. Progresses in sheet hydroforming, press forging and electromagnetic forming of sheet metal parts are also summarized.
\end{abstract}

KEY WORDS: Sheet forming; Crystal plasticity; Constitutive modeling; Mg alloy; UHSS; Hot stamping; Hydroforming; Aluminum alloy

\section{Introduction}

Sheet metals possess a non-substitutable role in the industry of electronics, automobile, aerospace, etc., and its forming technologies are also important to meet the increasing demand of better quality and more complex parts. Due to the above reasons, many new sheet metal forming technologies have been proposed during recent years in China, such as solid granular medium forming,

Available online at http://link.springer.com/journal/40195

Shi-Hong Zhang

shzhang@imr.ac.cn

1 Institute of Metal Research, Chinese Academy of Sciences, Shenyang 110016, China hydromechanical deep drawing, press forging, electromagnetic forming and hot stamping of UHSS. Unlike the conventional forming methods, these new technologies highlight the features of great flexibility, high efficiency and integrated forming. An example is hot stamping technology combining the forming process with quench and partition, which produces high-strength parts and saves costs simultaneously.

On the other hand, numerical methods have been proved a sufficient tool to optimize the forming parameters and can tackle the boundary conditions that are difficult to be imposed during experiments. Crystal plasticity method can capture the internal intrinsic deformation mechanism of grains to solve crystal mechanical problems during the simulation of sheet forming. It also offer great flexibility with respect to including texture evolution and various constitutive formulations for plastic flow, for instance, the 
twinning and detwinning process and their effects on the material texture and hardening behavior. Compared to crystal plasticity, the macroscopic phenomenal constitutive model is prone to be implemented into software and can describe complex deformation process during a reasonable cost and time. The constitutive models can enhance their ability by modifying the anisotropic parameters and/or updating the parameters though crystal plasticity.

This article reviewing the main developments of new technologies and theory of sheet forming can be divided into four sections.

\section{Advanced Constitutive Models of Sheet Metal Forming}

\subsection{Crystal Plasticity Method}

Crystal plasticity method considering crystallographic slipping, twinning, texture and its evolution is prone to describe the physical essence of plastic deformation. At present, the crystal plasticity theory can be divided into two types, Taylor model [1] and the self-consistent (SC) model $[2,3]$. Taylor model is relatively simple and easy to be implemented numerically, but this model does not consider the interaction between grains, and cannot describe the inhomogeneous deformation caused by anisotropy [4]. The self-consistent (SC) model based on mesoscopic inclusion theory possesses physical superiority to deal with twinning problems, and has developed the elasto-plastic self-consistent model (EPSC), visco-plastic self-consistent model (VPSC) and elasto-visco-plastic self-consistent model (EVPSC), which are suitable for small deformation, large deformation, creep behavior and other deformation process. Another method is to incorporate crystal plasticity model into the finite element software, named crystal plasticity finite element method (CPFEM). This method can be used to simulate deformation processes with the complicated boundary conditions.

With the development of computer technology, crystal plasticity theory has been widely used in the simulation of stamping forming process of sheet metal. Zhang et al. [5] developed a rate-related crystal plasticity model and studied the effect of different initial textures on deep drawing earing of face-centered cubic structure (FCC) sheet metal. Geng et al. [6] analyzed the non-homogenous microdeep drawing process of the polycrystalline copper, which indicates the effectiveness of CPFEM on the analysis of size effect during microstamping. The increasing of grain number weakens the non-homogenization leading to the decrease in earing height and an enhanced interaction between grains. Recently, $\mathrm{Li}$ et al. [7] adopt crystal plasticity theory dominated by slip and twinning which is suited for hexagonal close-packed (HCP) structural metal to investigate the deep drawing process of $\mathrm{AZ31} \mathrm{Mg}$ alloy. The twinning process is considered with predominant twin reorientation (PTR) model to deflect the matrix [8]. Plastic strain of the $\varepsilon^{p}$ in a single grain can be expressed by the following formula:

$\Delta \varepsilon^{p}=\left(1-\sum_{\beta=1}^{N^{w}} g^{\beta}\right) \sum_{\alpha=1}^{N^{s}} \Delta \gamma^{\alpha} \mathbf{p}^{\alpha}+\sum_{\beta=1}^{N^{t w}} g^{\beta} \Delta \gamma^{\beta} \mathbf{p}^{\beta}$,

where $N^{S}$ and $N^{t w}$ are the total number of activated slip system and twin variant, respectively, $\sum g^{\beta}$ is the twinning fraction, $\Delta \gamma^{\alpha}$ and $\Delta \gamma^{\beta}$ are the increment plastic strain of slip and twinning, respectively, and $\mathbf{P}$ is Schmid tensor.

As the initial conditions of finite element model, a group of Euler angle $(\Phi 1, \Phi, \Phi 2)$ of AZ31 rolled sheet is assigned to the polycrystal through the VUMAT subroutine. Figure 1 shows the simulation results of an AZ31 sheet metal cup. From Fig. 1a, the cup shows $45^{\circ}$ earing which is a significant deviation from the rolling direction, and Fig. $1 \mathrm{~b}$ shows that the texture with a significant (00001) (1010) and tensile twin (TT) components can be measured in the cup. From the experiment results in Figs. 2 and 3, the AZ31 cup also shows a $45^{\circ}$ ear, the same texture with

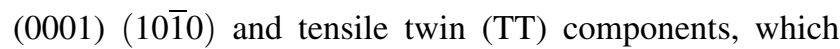
indicates the simulation of stamping cup ear contour and the texture is in good agreement with the experimental results. Crystal plasticity finite element simulation (CPFEM) can predict the deformation mechanism of $\mathrm{Mg}$ alloy parts formed by stamping very well.
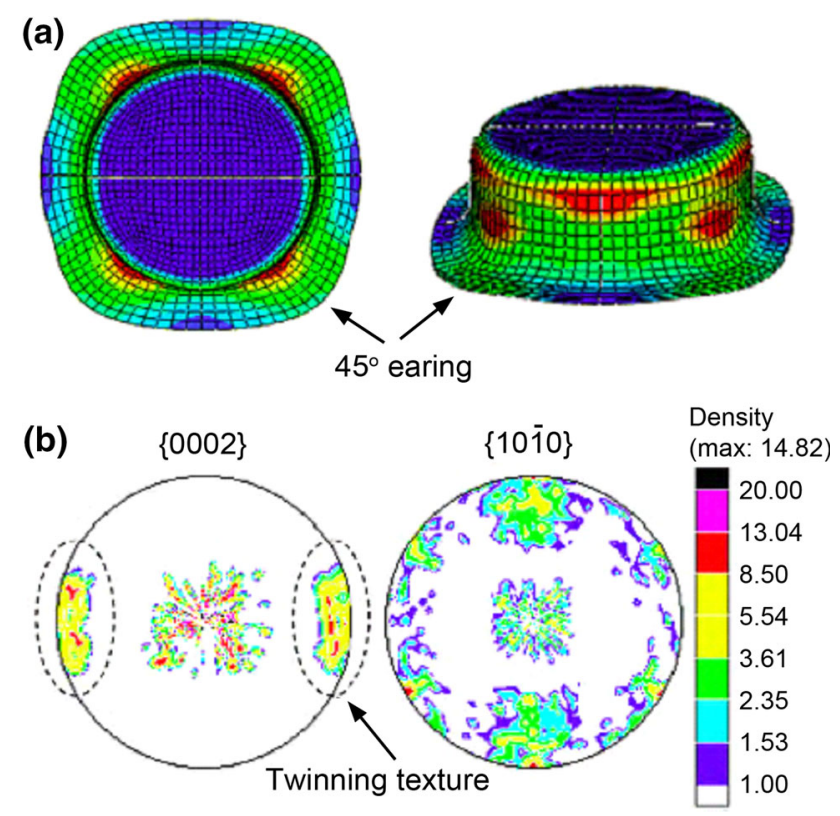

Fig. 1 Simulation results for deep-drawn cup of AZ31 alloy sheet formed at $150{ }^{\circ} \mathrm{C}$ : a earing profiles and $\mathbf{b}(10 \overline{1} 0)$ pole figures 

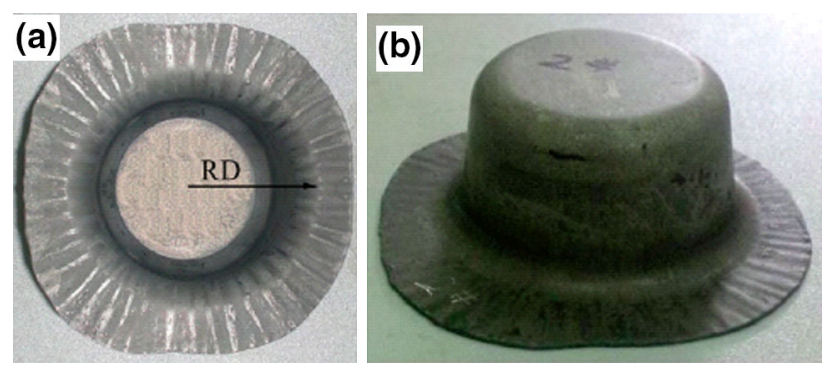

Fig. 2 Experimental results for deep-drawn cup of AZ31 alloy sheet formed at $150{ }^{\circ} \mathrm{C}$ : a photograph of bottom; b whole photograph

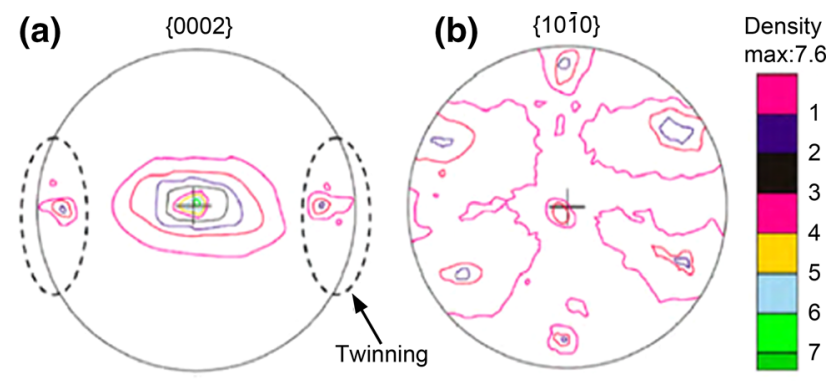

Fig. 3 Measured texture for deep-drawn cups of AZ31 alloy sheet: $\{0001\}$ pole figure; $\mathbf{b}$ pole figure

The twining and detwinning process can be observed under certain loading path (cyclic loading), which distinctly effects the deformation behavior of $\mathrm{Mg}$ alloy [9]. Zheng et al. [10-12] analyzed the detwinning behavior in the cyclic loading process with different pre-compressive strain, and revealed that detwinning similar to the twinning process is also mainly governed by the Schmid factor (SF). A new crystal plasticity model is established to depict the detwinning process, which is shown as follows:

$\tau_{\text {eff }}^{(\alpha)}=\tau^{(\alpha)}-\tau_{\text {kin }}^{(\alpha)}-\left|\tau_{u}^{(\alpha)}-\tau_{r}^{(\alpha)}\right|$,

where $\tau_{r}^{(\alpha)}$ is the resolved stress (RSS) before the grain rotation and $\tau_{u}^{(\alpha)}$ is the resolved stress at the unloading point of the compression. $\tau_{\text {kin }}^{(\alpha)}$ is the back stress, and the item $\left|\tau_{u}^{(\alpha)}-\tau_{r}^{(\alpha)}\right|$ is added to eliminate the effect of second elastic deformation during the compression process on the resolved stress of the slip during the inverse unloading process. Figure 4 shows the experiment and simulation results of the stress-strain curves along the rolling direction (RD), which suggests that the crystal plasticity model including the twinning and detwinning can describe the cyclic deformation of $\mathrm{Mg}$ alloy reasonably.

Crystal plasticity method lays a bridge of the gap between the polycrystalline texture and macroscopic forming ability of sheet metal, and gives an effective path to a more profound consideration of sheet metal anisotropy

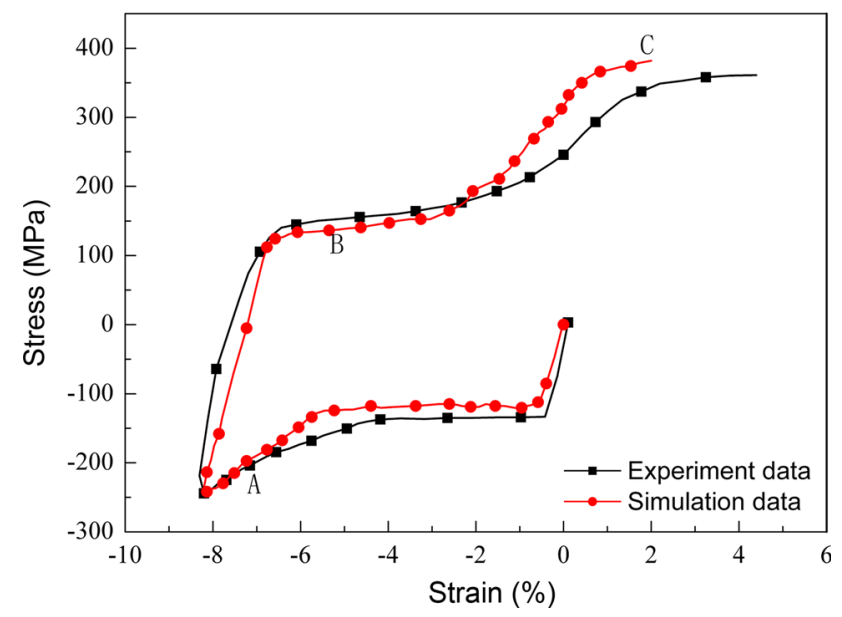

Fig. 4 Stress-strain curves of AZ31magnesium rolling sheet under compression-tension loading along RD (with pre-compression strain of $8 \%$ )

in their forming process simulations. It is also a great tool to investigate the unique deformation mechanism under some loading paths.

\subsection{Macroscopic Constitutive Model}

Although advantages of the crystal plasticity described above, the existing numerical and software associated with the CPFEM method need complex iteration process to solve amounts of nonlinear constitutive equations, which have the issues to improve convergence behavior of the CPFEM constitutive equations as well as a robust, modular and parallelized calculation method. Moreover, the sheet metals deformed with different strain paths may exhibit tension/compression asymmetry, crossing hardening effect, Bauschinger effect and hardening stagnation effect, which has a significant influence on the forming process. Compared to the crystal plasticity, the macroscopic constitutive equation can describe the above mechanical behaviors with a relatively simple formula and solution procedure with considering strain paths by different hardening models.

He et al. $[13,14]$ construct a new hierarchical multiscale model (HMS) based on the research of von Houtte et al. [15] to implant the texture evolution and anisotropic changes induced by twining into the macroconstitutive equation. In the new HMS, the macroscale finite element model is used to simulate the forming process, and texture evolution is calculated by the visco-plastic self-consistent (VPSC) model. Meanwhile, it should be noticed that the calculation is not carried out at each integration point of each incremental step, which is only updated when certain criteria are satisfied. The author combines the modified anisotropy and asymmetry of tension and compression yield equation (ECPBt2), enhanced hardening model with 
strain path changes (EHAH) and visco-plastic self-consistent model (VPSC). Simulation of the process of deep drawing forming of TA1 cylinder is carried out by using the ECPBt2-VPSC model, ECPBt2-EHAH model, ECPBt2-HMS-EHAH model, separately, and the results are shown in Fig. 5a-c. Figure 6 shows a typical TA1 cup with obvious ears after cup drawing.

Form the wall height in Fig. 7, it can be seen that wall height predicted by the ECPBt2-HMS-EHAH model considering the texture evolution and strain path changes shows a reasonable agreement with the experimental data. The constitutive model can accurately describe the deformation behavior of TA1. Use of the HMS model can lead to the effective combination of macro- and microsimulation and significantly improves the prediction accuracy of earing and springback of stamping parts.

Lang et al. [16] establishe the constitutive equation of 5A06-O aluminum-magnesium $(\mathrm{Al}-\mathrm{Mg})$ alloy under warm conditions by increasing function subtraction method. The constitutive equation established by this method has a simple form and could reflect the influence of temperature and strain rate and describe the complex flow stress-strain curve more accurately. Non-saturating kinematic (NSK) Swift model based on the principle of energy dissipation was proposed by Chen et al. [17, 18] to describe the cyclic loading permanent softening effect. As shown in Figs. 8 and 9, the springback prediction of the Numisheet'05 example shows a good agreement with the experimental measurement. In addition, nice agreements are also achieved between the measured data and the numerical results of the springback and the thickness prediction for two DP sheet metals (DP600 and DP965). Both the theoretical importance of the control functions in material modeling and practical significance of the obtained nonsaturating kinematic (NSK) Swift model can be implied through the constitutive verification and the industrial application.

In summary, the effect of strain path is the main focus of the macroscopic constitutive model. The multi-scale model based on physical mechanism is an effective method to improve the prediction accuracy of sheet metal deformation under different strain paths.

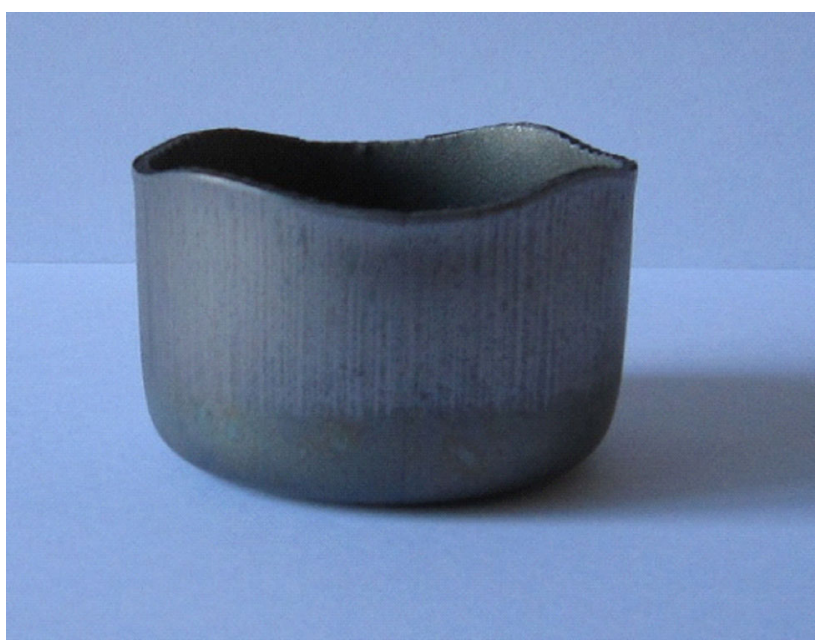

Fig. 6 Photograph of typical TA1 cup after cup-drawing

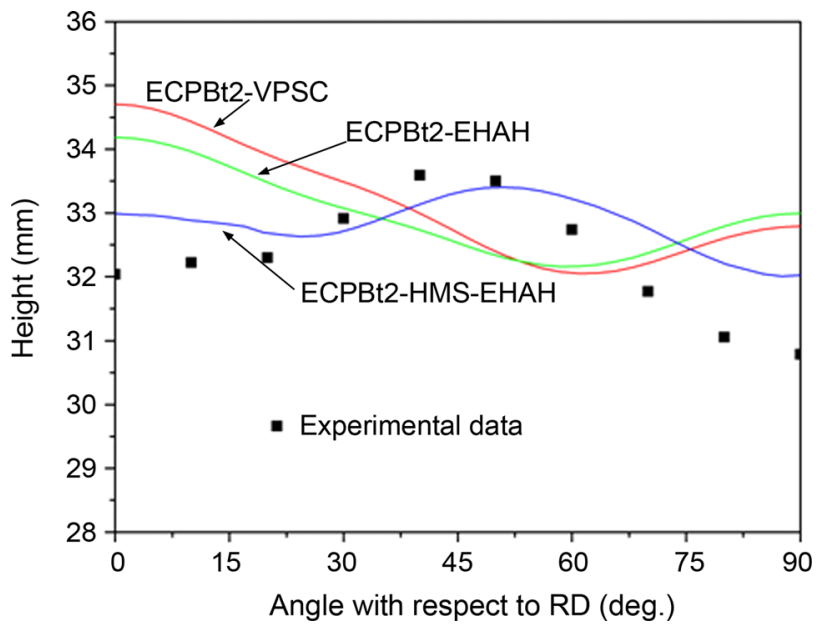

Fig. 7 Experimental and predicated wall height by ECPBt2-VPSC model, ECPBt2-EHAH model and ECPBt2-HMS-EHAH model

\section{Experimental and Theoretical Prediction of Forming Limit of Sheet Metal}

The formability of sheet metal is generally accepted to be evaluated by the so-called forming limit curve (FLC), and its main advantage is the easy interpretation of simulation (a)

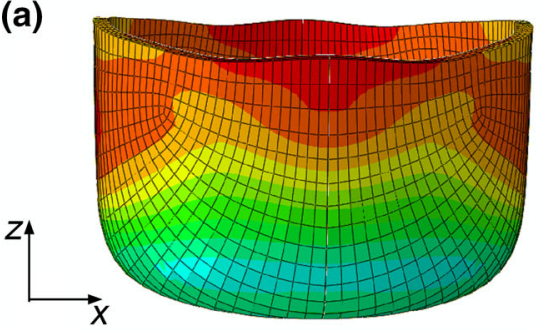

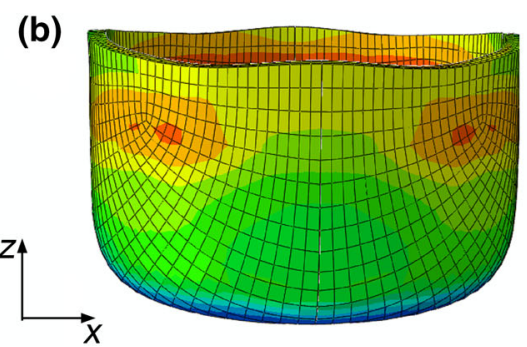



Fig. 5 Predicated earings for anisotropic cups obtained with different constitutive models: a ECPBt2-VPSC model; b ECPBt2-EHAH model; and c ECPBt2-HMS-EHAH model (update strain 0.08) 


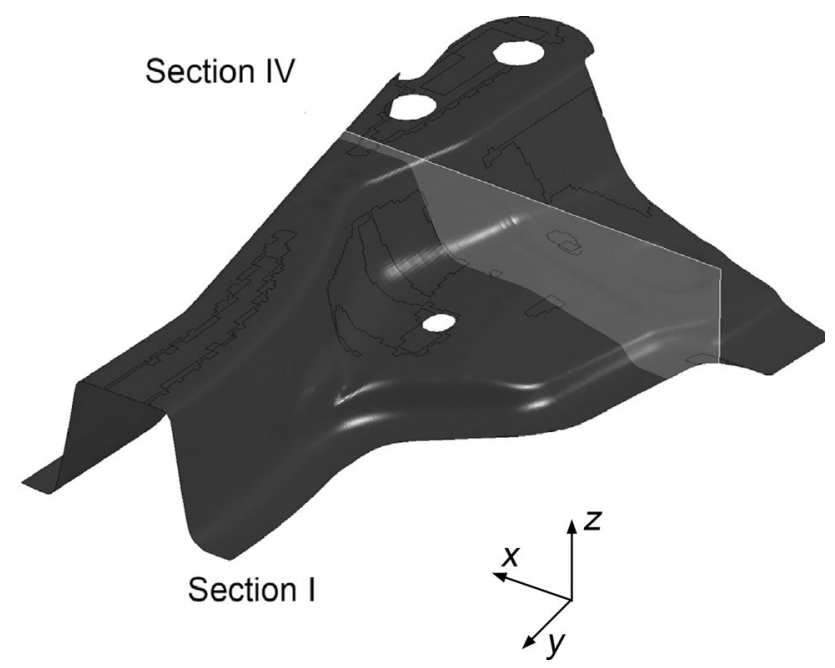

Fig. 8 Sections specified by Benchmark 2 of Numisheet'05 (Section I at $y=0 \mathrm{~mm}$ and Section IV at $y=-370 \mathrm{~mm}$ )

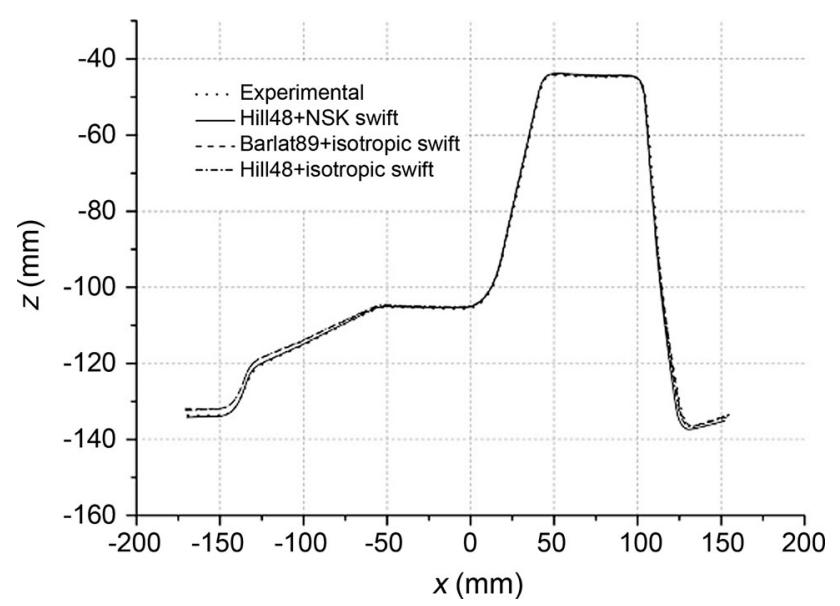

Fig. 9 Comparison between experimental data and simulation results of springback profiles at Section IV (DP600, Benchmark 2 of Numisheet'05)

or experimental data. The traditional FLC is usually determined by failure criterion or experimental method.

The experimental research mainly focuses on FLC of several hard forming materials under warm condition. The results of Ma et al. [19] show that the FLC of 5A90 Al-Li alloy increases with a higher temperature. Furthermore, the FLC calculating model of $5 \mathrm{~A} 90 \mathrm{Al}-\mathrm{Li}$ alloy is built, which is the important foundation to predict the FLC of $\mathrm{Al}-\mathrm{Li}$ alloy at any temperature. Huang [20] made a good prediction of FLC of 7075-T6 Al alloy sheet with ductile fracture criterion considering temperature effect. Zhong et al. [21] showed that the FLC of AZ31B Mg alloy sheet increased with the temperature rising from 150 to $250{ }^{\circ} \mathrm{C}$. The above results indicate that the temperature plays an important role on the improved formability of sheet metals.
The failure criterion on the forming limit is divided into the following two categories: The first one is the classical bifurcation theory, such as Swift's diffuse necking model [22], Hill's localized necking model [23] and Rice [24, 25], and the second one is the hypothesis based on the initial local inhomogeneity, such as M-K theory [26] (see in Fig. 10). Now, the majority of the theoretical study on the forming limit is based on the $\mathrm{M}-\mathrm{K}$ theory taking into account different advanced constitutive equations with the cumulative damage model.

Du et al. [27] systematically investigated the effect of material parameters, such as strain hardening index $n$, strain rate sensitivity index $m$, prestrain $\varepsilon$ and temperature $T$, on the forming limit curve of 5A90 Al- $\mathrm{Li}$ alloy and TRIP steels based on M-K theory. Meanwhile, it indicates that the theoretical predictions are closer to the experimental data by using modified initial imperfection factor.

Lang et al. [28] analyzed the influences of normal pressure and temperature on the forming limit during the warm hydroforming process. Figures 11 and 12 show that the forming limit will be improved with the rising of normal forming pressure and temperature. The increase in FLC is formulized in a full quadratic form, which is a function of temperature and through thickness normal stress. The left quadrant of FLD was determined by the cylindrical warm hydromechanical cup deep drawing, which shows a good accordance with the predicted results by using the modified $\mathrm{M}-\mathrm{K}$ model.

Fang et al. [29] compare the yield functions (Hill's 48, Hill's 79 and non-quadric Hosford) and their effect on the stress calculation of the translation from the FLD to FLSD, which suggests that the Voce hardening law and the Hosford yield criterion are appropriate for the FLSD calculation of the Al alloy 1060. Yang et al. [30] combined the classic M-K theory and the ductile fracture criteria $(\mathrm{C}-\mathrm{L})$ proposed by Cockcroft and Latham [31], and the control equation is shown as follows:

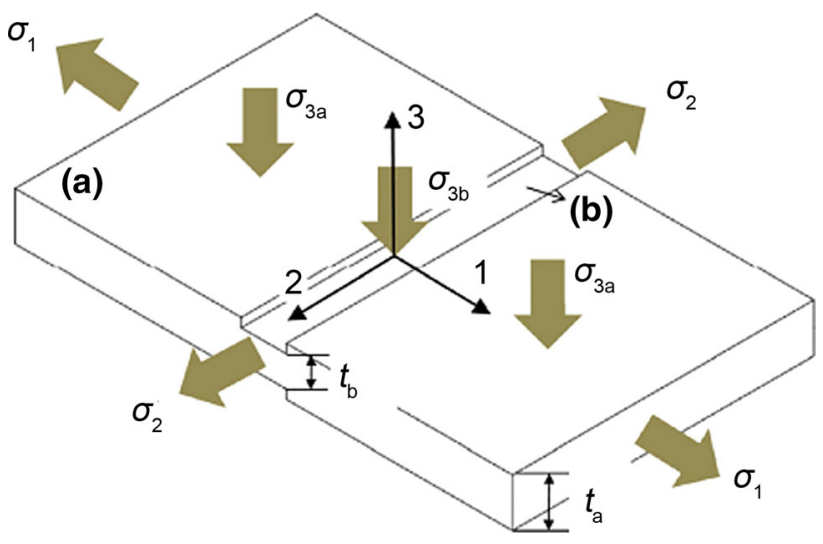

Fig. 10 Theoretical frame diagram of M-K model 


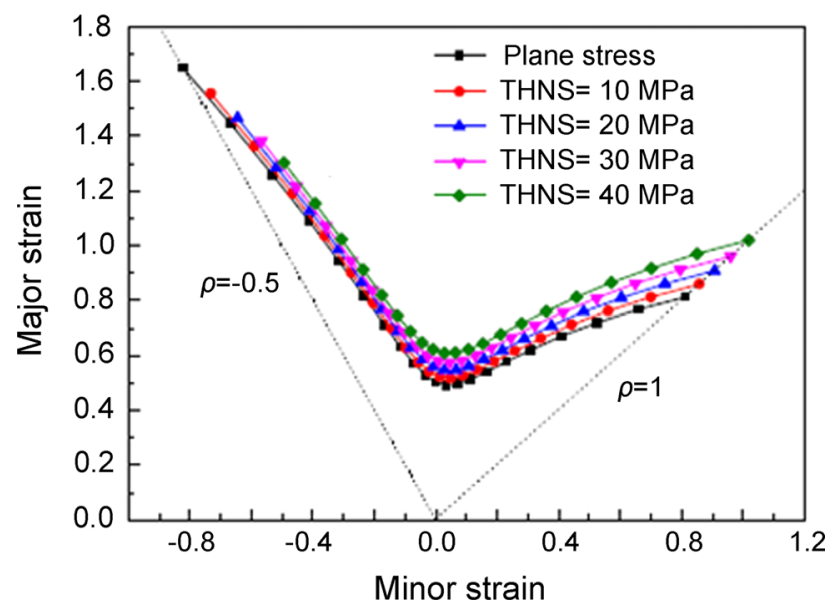

Fig. 11 Influence of through thickness normal stress on FLD for 5A06, where $f_{0}=0.995, T=200{ }^{\circ} \mathrm{C}, r=1.0$

$\int_{0}^{\overline{\varepsilon_{f}}} f\left(\bar{\sigma}, \sigma_{m}, \ldots\right) d \bar{\varepsilon}=C$,

where $\int_{0}^{\overline{f_{f}}} f\left(\bar{\sigma}, \sigma_{m}, \ldots\right)$ is the yield function, $\bar{\varepsilon}$ is the equivalent strain and $C$ is the material parameter. A good prediction of forming limit of AA7075-O can be achieved by considering the strain path changes with Hill's 48 anisotropy yield function and modified Swift hardening law.

Chen et al. [32] point out the uncertainty of $f_{0}$ in $\mathrm{M}-\mathrm{K}$ theory and inaccuracy of the ductile facture criteria. A new forming criterion is proposed by modification of $\mathrm{C}-\mathrm{L}$ model and considering the strain paths, which is expressed as follows:

$\int_{0}^{\overline{\varepsilon_{n}}} \bar{\sigma} d \bar{\varepsilon}=w \cdot\left[(1+|\alpha|)^{p}-Q\right]$,

where $\alpha=\Delta \varepsilon_{2} / \Delta \varepsilon_{1}$ and parameters $P$ and $Q$ are related to the deformation mode and material properties. The calculation, as well as the experimental results, shows that the criterion can predict forming limits for steel and aluminum sheets exactly and makes it possible to determine forming limit strains under different strain paths only through tensile test.

The above experimental works [18-21] and calculation of failure criterion [27-29] are all conducted under the linear strain paths, i.e., the deformation histories with nonlinear strain paths and their distinct effects on the FLC are neglected. Otherwise, the results $[30,32]$ indicate that the combination of the classic $\mathrm{M}-\mathrm{K}$ theory and the ductile fracture criteria $(\mathrm{C}-\mathrm{L})$ is an efficient way to conquer the drawbacks of $\mathrm{M}-\mathrm{K}$ theory, and more attention should be paid to the failure criterion taking into the strain path effects in the future research,

\section{New Technologies for Sheet Metal Forming}

\subsection{Sheet Forming with Flexible Medium Assistance}

\subsubsection{Solid Granular Medium Forming}

As a novel technology for sheet forming, the apparent difference between solid granular medium forming (SGMF) and the existing flexible forming methods is that solid granular medium changes technology implementation and forming mechanism. SGMF shown in Fig. 13 adopts the spherical metal or nonmetal particles with diameter in the range of $0.05-2.00 \mathrm{~mm}$ as filling medium; it has the advantages of good filling capacity, flow property, chemical stability and no corrosion on artifacts. The particles medium can overcome the sealing and loading induced by liquid media under the high-temperature condition.
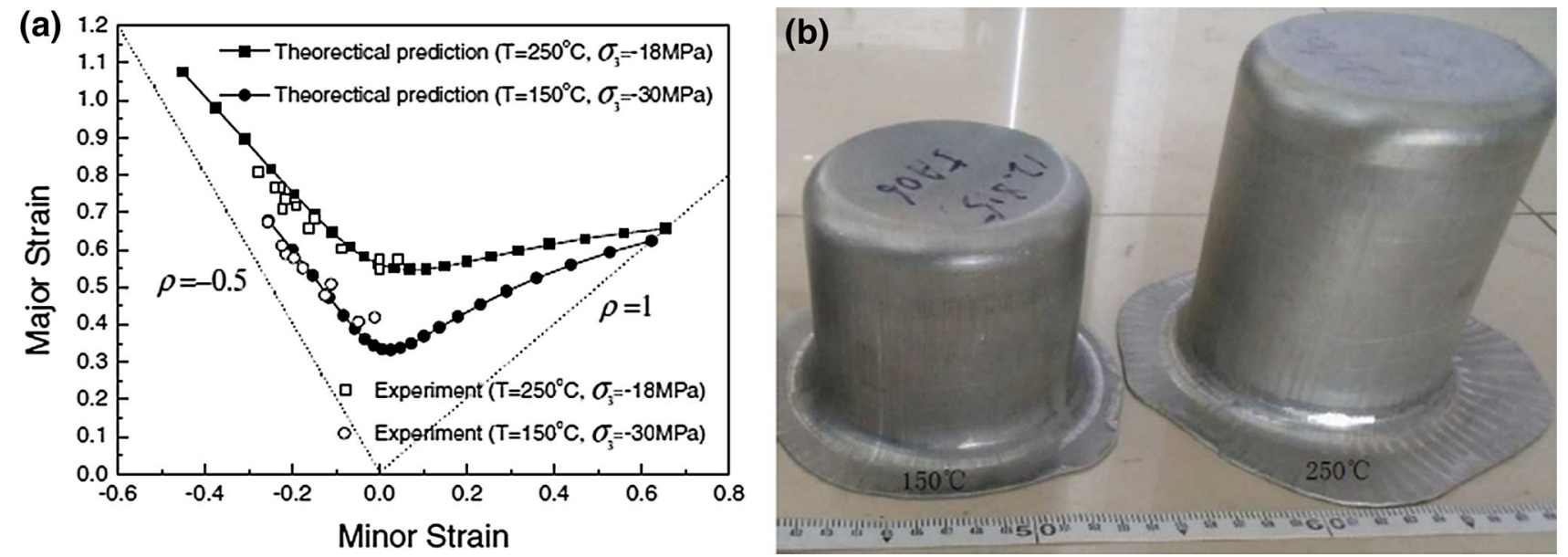

Fig. 12 Warm hydromechanical deep drawing for 5A06 aluminum alloy: a comparison of theoretical prediction with experiment results in the left quadrant of FLD and $\mathbf{b}$ the hydroformed parts in experiment with carved crossing square grids 


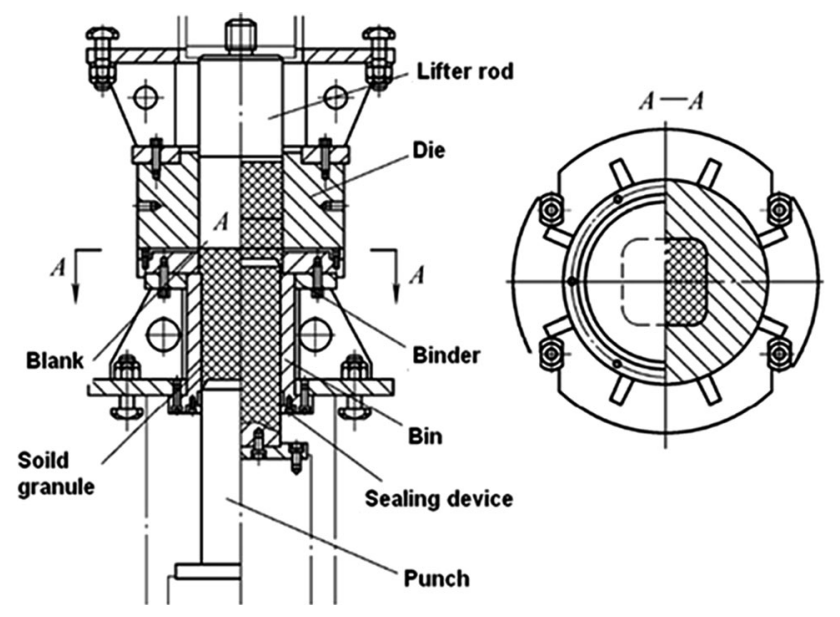

Fig. 13 Solid granular medium forming process

Moreover, friction produced by the granular medium adhering to the sheet surface results in uniform wall thickness of components. SGMF provides a new method for the process of material fabrication and possesses a wide application prospect due to its advantages of simple process implementation, good surface quality, better die fittingness, high dimension precision [33].

Cao et al. [34] analyze the distribution of granular medium forming and granular medium force transmission characteristics under high pressure by using the experimental device shown in Fig. 14. Particle body pressure head of compressive stress is $\sigma$, the bottom reaction stress is $\sigma_{u}$, and the radial stress on the side wall is $\sigma_{r}$.

Studies of the data acquisition show that the stressstrain relationships of the granular system are obviously nonlinear which can be well represented precisely by a power function formula:

$\varepsilon=9.86 \times 10^{-3} \sigma^{0.335}$,

where $\varepsilon$ is the axial strain of medium particles. Meanwhile, discrete/distinct element method (DEM) is adapted to calculate changes in the load situation during the particle gravity stacking and the subsequent compression process. With load increasing, it can be known from Fig. 15 that the sub-rounded force chain network is gradually evolved into ellipse (Fig. 15d). The particles are gradually compacted and tend to be dense. Force chain network of particles system presents the regular fibrous weave, which attenuate with the increase in distance to punch.

Dong et al. [35] used an extended Drucker-Prager linear model in powder mechanics to construct finite element model of SGMF. Drucker-Prager linear model is shown as:

$F=t-p \tan \beta-c=0$,

where $p$ is the average principal stress, $c$ is cohesive force of material, for the non-viscous granular material $c=0, \beta$

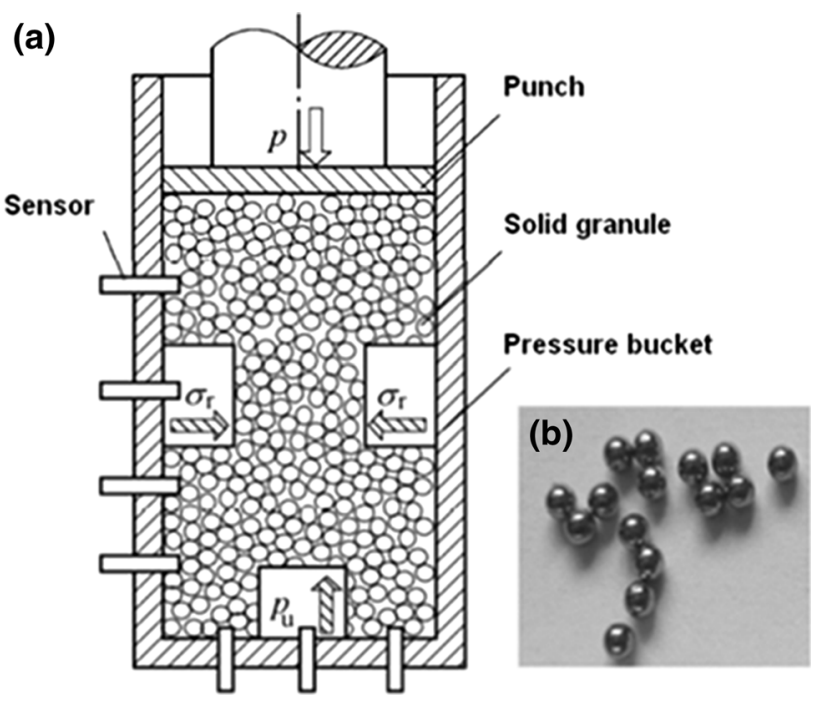

Fig. 14 Solid granule medium and the pressure test schematic diagram: a schematic of pressure test; $\mathbf{b}$ solid medium

is GM particle linear yield stress in the plane Angle, called as friction angle of Drucker-Prager model and $t$ is deviatoric stress parameter that expresses the roundness of the yield surface on the plane of $\pi$, as shown below:

$t=\frac{1}{2}\left[1+\frac{1}{k}-\left(1-\frac{1}{k}\right)\left(\frac{r}{q}\right)^{3}\right]$,

where $k$ is the ratio of triaxial tensile yield stress to triaxial compression yield stress, which controls the dependence of the yield surface on the intermediate principal stress value, $q$ is equivalent shear stress and $\mathrm{r}$ is the third stress invariant. The relationship of normal stress $\sigma$ and shear stress $\tau$ for different particle diameters is obtained by friction performance test, which agrees with hypothesis of Mohr-Coulomb yield criterion shown below:

$\tau=c-\sigma \tan \varphi$.

Loading curves, deformation characteristics in forming process and wall thickness distribution curves are proved in basic agreement with the result of finite element simulation. Studies show that the deformation characteristics and the force parameters which are close to the experiments can be generated from the numerical analysis on the SGM material model based on powder mechanic. Figure 16a, b shows the boxlike parts at different forming stages and the final formed parts.

In addition, Cao et al. [36] studies influence the temperature effect on deep drawing of AZ31B Mg alloy sheet by SMFG. The result indicates that the sheet exhibits a better drawing performance with the sheet temperature range of $250-300{ }^{\circ} \mathrm{C}$ and granular medium of $100-150{ }^{\circ} \mathrm{C}$, and the LDR can reach 2.4 or above. Recently, Cao et al. 


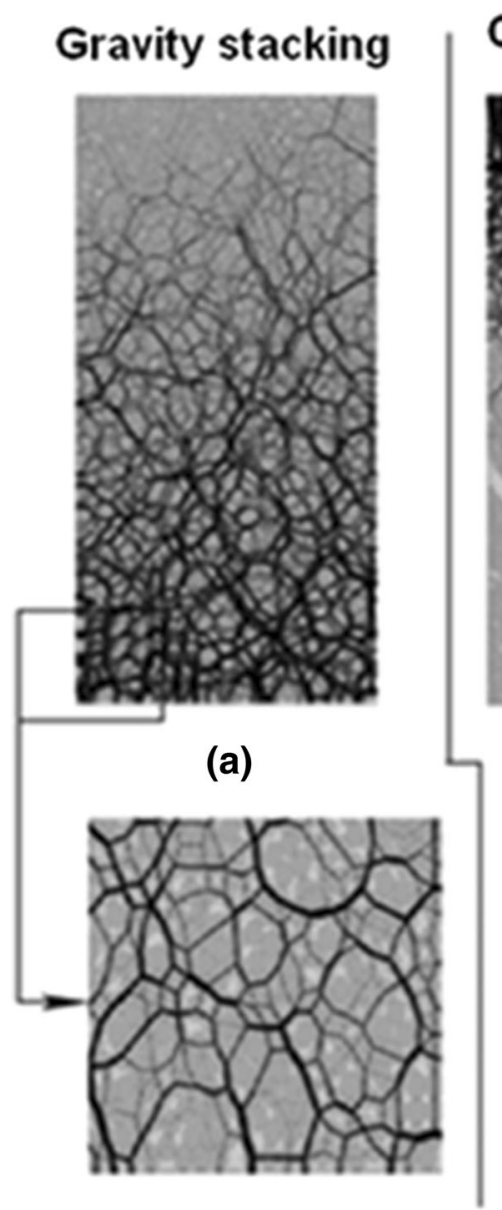

(e)

\section{Compression}

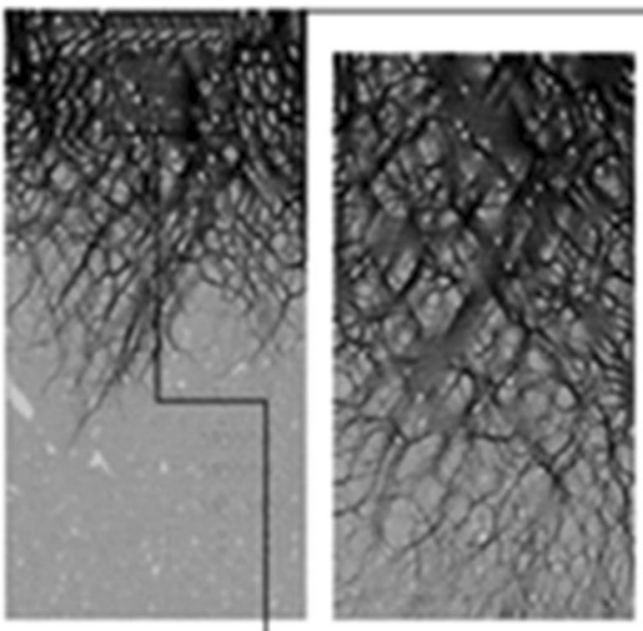

(c)

(b)

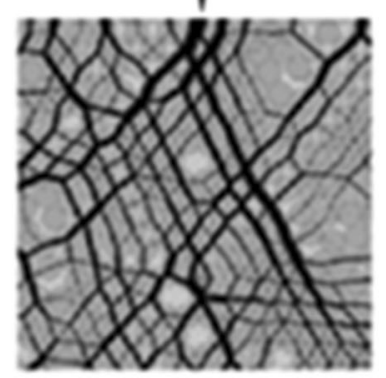

(f)

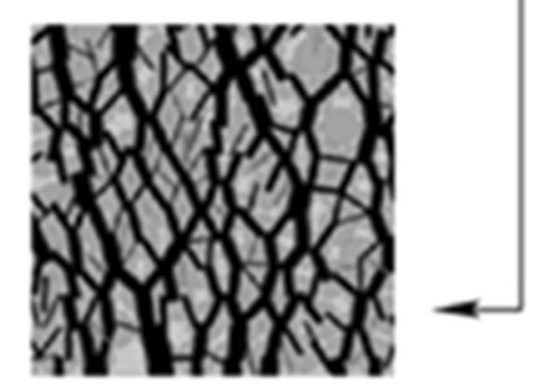

(g)

Fig. 15 Different stages of force chain network configuration of particle system
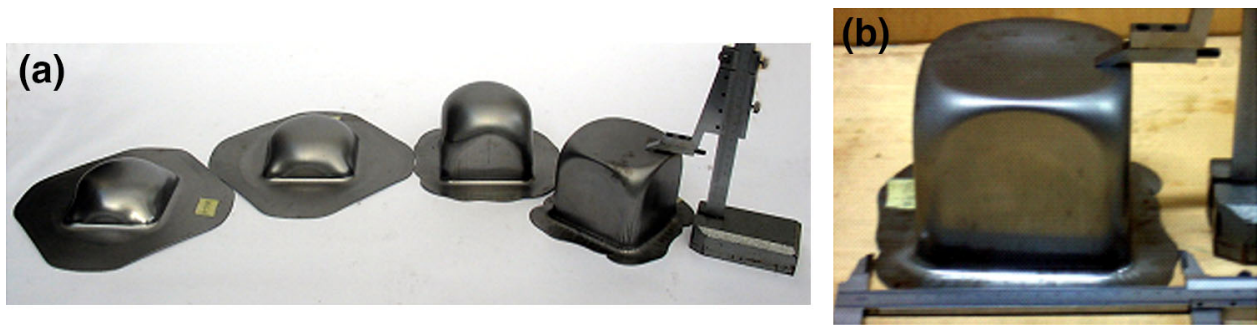

Fig. 16 Square box formed by SMFG: a forming stages of boxlike part; b boxlike part

[37] obtained the average punch pressure through the mechanical analysis of straight wall of the part based on the attenuation model in solid particles force. Obviously, the rise in friction between sheet and die surface will lead to increase in punch pressure.

Besides the above results, some researchers have extended this method to the tube bending, which is used to produce the super thin and large bending-angle tubes. At present, the traditional manufacture technologies of bending tubes are rotary bending, press bending and push bending, etc. Rotary bending is the most commonly used method for pipe bending. However, it is limited when used with regard to relative bending radius $(R / D)$ and the relative thickness $(t / D)$, and also needs mandrel, wiper die, clamp die, etc., which increases the complexity of the process. Similarly, press bending method for super large diameter and thin-walled tubes has difficulty to avoid external fracture and internal wrinkling. With the decreasing in relative bending radius $(R / D)$ and the relative thickness $(t / D)$ of tubes, forming defects are prone to be caused, for example thickness thinning in external wall, wrinkling and thickness thickening in internal wall, cross 
section distortion and springback. Most research work on bending tubes is concentrated on tubes with wall thickness higher than $1 \mathrm{~mm}$ and the ratio $t / D$ larger than 0.06 . However, there are almost no research literatures about the bending tubes with $t / D$ smaller than 0.06 , because it is difficult to form even with the advanced numerical control rotary bending machine [38, 39].

Thin-walled tubes were successfully produced by combing solid granular method with the die push bending process (see Fig. 17a), of which the relative bending radius $(R / D)$ is 1.5 and 3 , the bending angle is $90^{\circ}$ and $t / D$ is just 0.01 . The original tubes are $\Phi 30 \mathrm{~mm} \times 0.3 \mathrm{~mm}$ and $\Phi 70 \mathrm{~mm} \times 0.7 \mathrm{~mm}$. The final formed tubes are shown in Fig. 17b.

\subsubsection{HydroMechanical Deep Drawing of Sheet Metal Parts}

Hydromechanical deep drawing (HDD) combines the features of both traditional deep drawing technology and hydroforming technology [40].

Wang et al. [41] proposed a new numerical analysis method which couples finite element method and meshless method to analyze viscous pressure forming and sheet metal polyurethane rubber forming. The visco-elastoplastic constitutive model of viscous medium was set up. Serval key factors such as frictional contact state and adhesive stress modes between viscous medium and sheet metal were also studied. Based on the above theories, a coupled analysis model was developed to investigate flexible-die forming for sheet metal numerically. The validity of the predicted model is verified through experimental measurement. Wang et al. [42] investigated bulging deformation of sheet metal under variable viscosity of magnetorheological fluid produced by a designed bulging device, and force field and magnetic field can be applied simultaneously. With the electric current of magnetorheological fluid increasing, a higher bulging height and better strain distribution can be obtained.

Zhou et al. [43] have researched the wrinkle-free and anti-break limit criterion of cylindrical parts in the forming process of HDD, and gained security zone map of cylindrical parts with the method of HDD, which can provide the basic guideline for process parameters determination, die design and equipment selection. Chu et al. [44] used the local bifurcation theory as the failure criterion of forming limit for the prediction of wrinking and demonstrated that there is a strong dependency of the critical bifurcated stress at the onset of wrinkling on the shear stress imposed on the flange. The results in [45] show that static dent resistance is influenced by the deformation modes and equivalent effective strain of the formed part. When the strain ratio $\beta$ is in the range of $-1<\beta \leq 1$, the static dent resistance load is maximum, so there is an extreme value line of static dent resistance in 2D strain diagram shown in Fig. 18. Therefore, extreme value line of static dent resistance can be used for strain designing, corresponding to the best static dent resistance of the formed parts.

Lang et al. [46] analyzed the effect of maximum liquid pressure in die cavity and the gap of blank holder on the part quality. Further investigation about the effect of prebulging and backward bulging on the part forming indicates that the suitable pre-bulging and backward bulging can improve the quality of parts [47-49]. There are lots of research works about liquid pressure control and distribution aimed to change the stress state of the workpiece, such as double-side pressure [50-52], fluid-assisted blank holder deep drawing [53], radial-assisted pressure deep drawing [54-57] and inward flowing liquid deep drawing [58]. All the methods above can improve the forming ability of the material and the quality of part. Dong et al. [59] proposed the warm solid granular as drawing medium for sheet deep
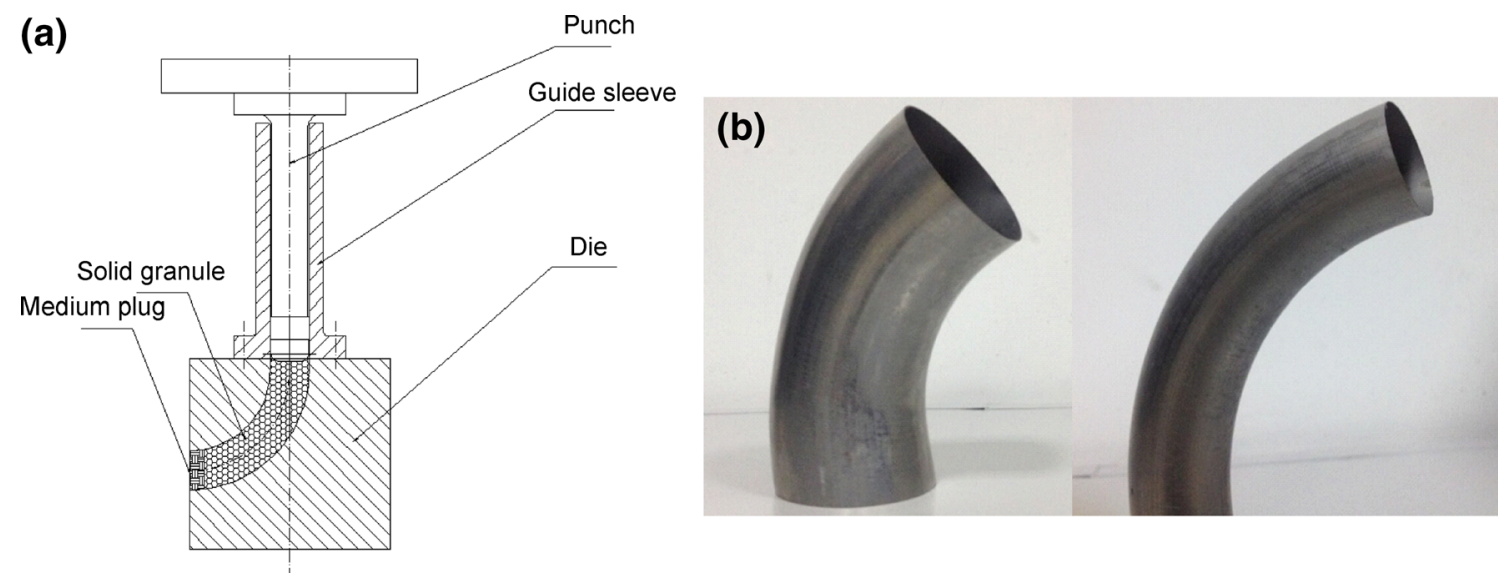

Fig. 17 Solid granule forming of small and large diameter thin-walled tubes: a schematic diagram of push bending process; $\mathbf{b}$ photographs of final formed tubes 


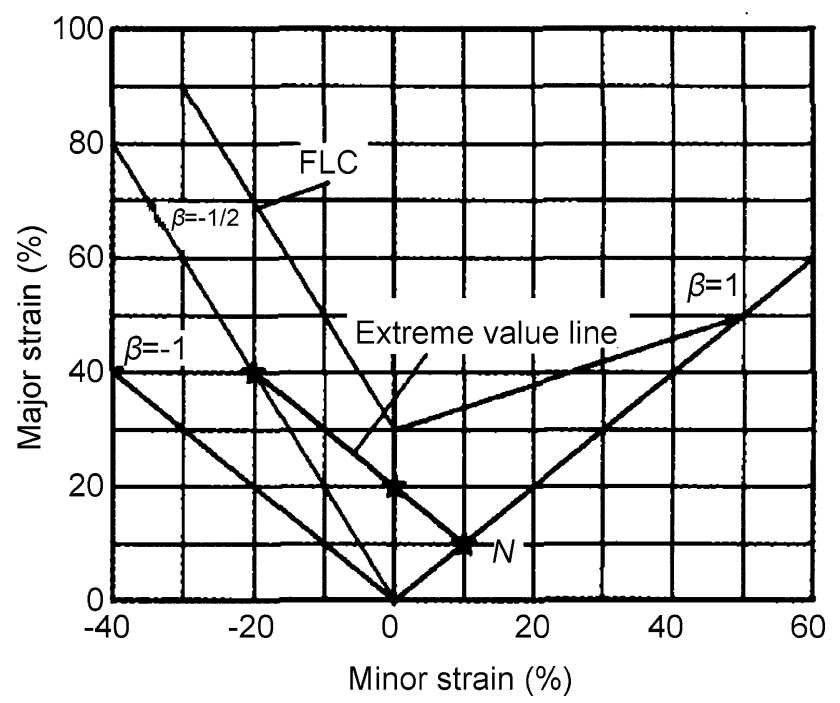

Fig. 18 FLC and extreme value line of static dent resistance of DP340/590

drawing to overcome the shortcomings of hydromechanical deep drawing that the liquid medium cannot withstand a high temperature. This technology solves the problems of loading and seal in the existing flexible-die forming process and provides a novel method for thermoforming of light alloy sheets, such as $\mathrm{Mg}$ alloys, $\mathrm{Al}$ alloys and $\mathrm{Ti}$ alloys. Jin et al. [60] researched segmented binder with variable blank holding force, which increases the forming accuracy and thickness uniformity and decreases the thickness reduction. Ding et al. [61] found that reverse hydromechanical drawing can greatly improve the limit drawing ratio (LDR) of $\mathrm{Al}$ alloys and obtained 3.3 LDR part that common HDD cannot reach.

Zhu et al. [62] researched the forming of complicated thin-wall superalloy parts. Figure 19 shows the multistages HDD assisted by conventional deep drawing, and parts with uniform thickness can be successfully formed.

Meng et al. [63] researched the strategy for manufacturing complex parts which cannot be formed by conventional deep drawing. As shown in Fig. 20, wrinkling and fracture are investigated which are the main failure types in hydromechanical deep drawing process of stainless steel parts with complex surface. The results show that loading curve of the cavity pressure should be adjusted according to the changes in the areas without tool contact. In addition, appropriate draw beads have been set up to prevent inner wrinkles on the transition regions (see Fig. 21).

Tai et al. [64] found that the warm hydrodynamic deep drawing with gradient temperature field on the sheet can be used to increase the limit drawing ratio. Sun et al. [65] simulated the influence of blank holder force, drawing speed and friction coefficient on the deep drawing process

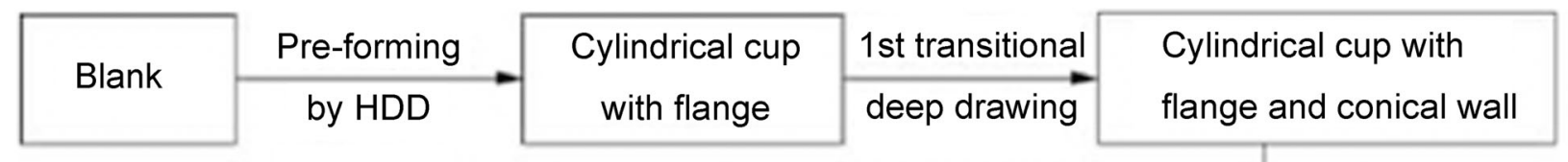

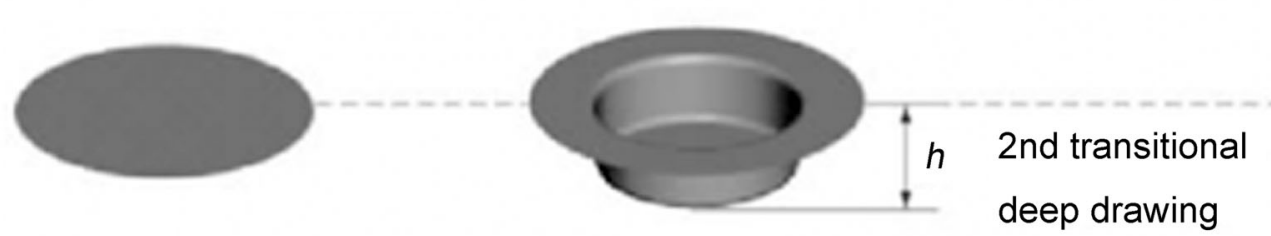

(a)

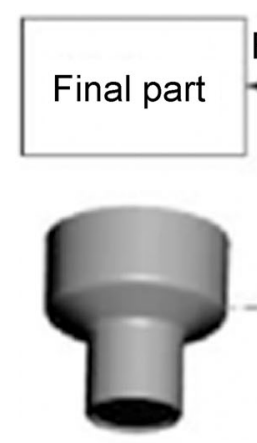

(f) (b)

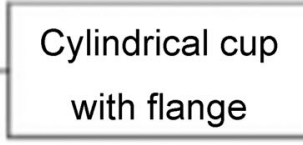

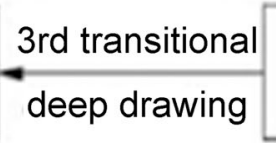

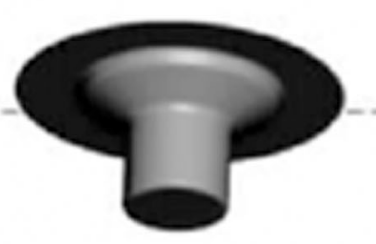

(e)

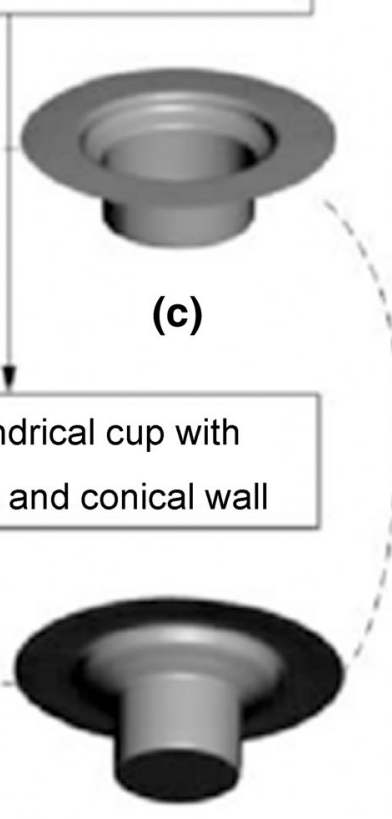

(d)

Fig. 19 Forming sequence of combination of HDD with traditional DD 



Fig. 20 Failures caused by draw beads: a crack by transverse draw beads; $\mathbf{b}$ crack by longitudinal draw beads; $\mathbf{c}$ wrinkle; $\mathbf{d}$ qualified part

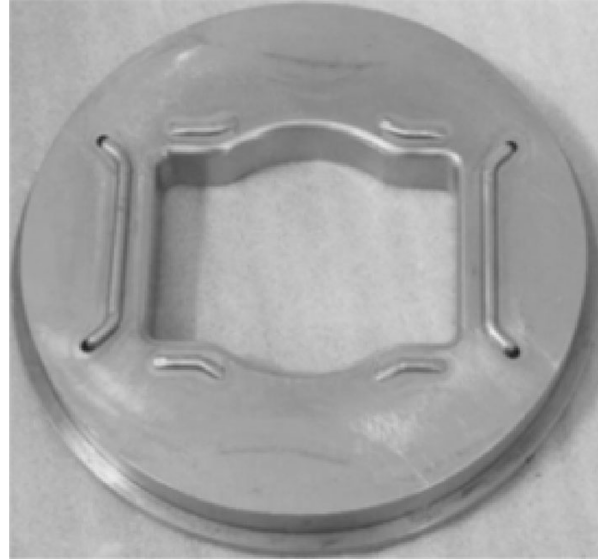

Fig. 21 Arrangement mode of draw beads

of sheet metal. Lang et al. [66] analyzed cone-shaped cups formed by active hydroforming and passive hydroforming, respectively, and proposed a new method of HDD where the cups pre-drawn by passive hydroforming are finally formed with the active hydroforming. Liu et al. [67] researched double-step cylinder deep drawing which can decrease the formation and thinning of impending area in the early period. The effects of the strain hardening exponent $n$ and the anisotropy coefficient $r$ on the HDD of the irregular cup are analyzed by Liu et al. [68], and the major strain and the thickness distribution are used as evaluation criteria. Fu et al. [69] researched the aluminum alloy automobile outdoor formed by HDD adopting variable blank holder force based on numerical simulation, and the results show that compared with common drawing process, parts quality has been greatly improved with small thickness thinning, a better forming precision and uniform thickness distribution.

The literatures above introduce the state of the art of HDD technology, and the new developing technologies, including viscous medium deep drawing, warm solid granular deep drawing and the evaluation criteria of material formability under HDD. The research of various process parameters, such as blank holder force, pre-bulging, double-side pressure, and radial-assisted pressure, is also presented in detail. With the developing demands of sheet metal parts with large size, thin wall, deep cavity, complicated curved surface and hard forming materials, the manufacturing industries, such as aviation, aerospace and automobile, have paid more attentions to the low-cost and high flexible HDD technology.

\subsection{Press Forging of Sheet Metals}

Local volume or press forging forming, which is a new hybrid plastic forming process comprising stamping and forging, can produce parts with a large surface area and thickness difference. It can realize the near-net forming and has great application in $3 \mathrm{C}$ electronic devices, automobile disk-type parts [70].

Zhang et al. [71] successfully made a series of 3C magnesium alloy electronic devices (Fig. 22) with the wall thickness of $0.6 \mathrm{~mm}$ and boss height of $4.5 \mathrm{~mm}$ by press forging, and analyzed the effect of friction on the forming pressure and quality through simulation and experimental research, which provides theoretical and experimental guideline for press forging of magnesium alloy parts. (a)

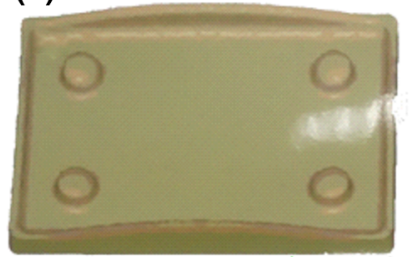

(c)

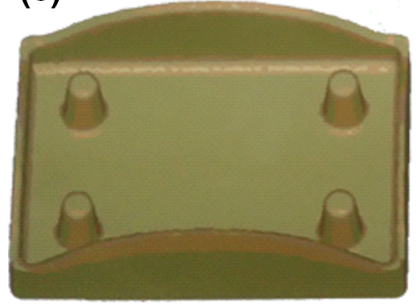

(b)

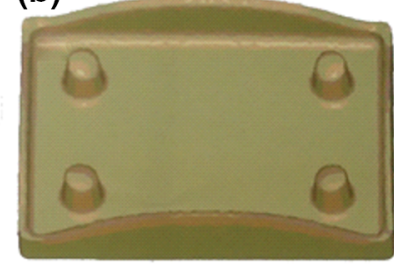

(d)

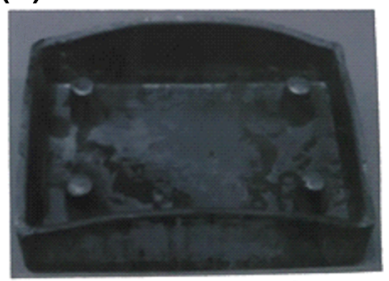

Fig. 22 Forming process (FEM) and experiment result: a stroke $0.6 \mathrm{~mm}$; b stroke $0.9 \mathrm{~mm}$; c stroke $1.2 \mathrm{~mm}$; d experimental part 


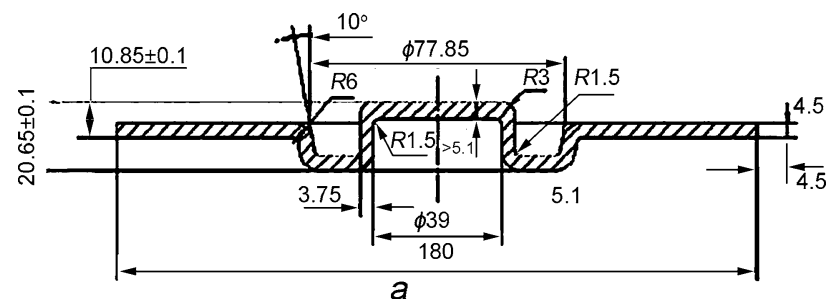

Fig. 23 Geometry model

Wang et al. [72-74] studied the warm press forging forming process of fly wheel and clutch hub body parts, suitably indicating that the autoparts with minimal radius can be prepared successfully by the reasonable forming process and die fillets. Instead of the traditional stampingwelding process, this technology can improve the production efficiency with the material using rate increasing from $45 \%$ to more than $85 \%$. Xue et al. [75] proposed the press forging hybrid forming for pulley parts with small inner and outer rounded corners and thickness difference in the horizontal and the vertical directions. Geometry and forming process of pulley parts are shown in Figs. 23 and 24 . The forming result is qualified without fold defect when the value of the lower fillet radius $R$ of the die is $10 \mathrm{~mm}$. The blocking effect is the best when the value of resistance bevel angle of wall is $45^{\circ}$.Through the rational design of intermediate thickening, die fillet and angle, the parts can be made with a good surface quality, and the final shape of the press forged parts is shown in Fig. 25.

The key step of press forging is a good die design in order to reach local thickening by the forming process. The authors above have a certain degree of innovation on the die design to adjust material flow in the forging process, and this provides a solid foundation for future development of the other parts.

\subsection{Electromagnetic Forming Technology}

Electromagnetic forming (EMF) is a kind of powerful and high-speed forming technique that the material is formed under the pulse electromagnetic force (PEF) induced by a pulse magnetic field. Since the electromagnetic force is generated instantly, the strain rate of sheet metal can reach

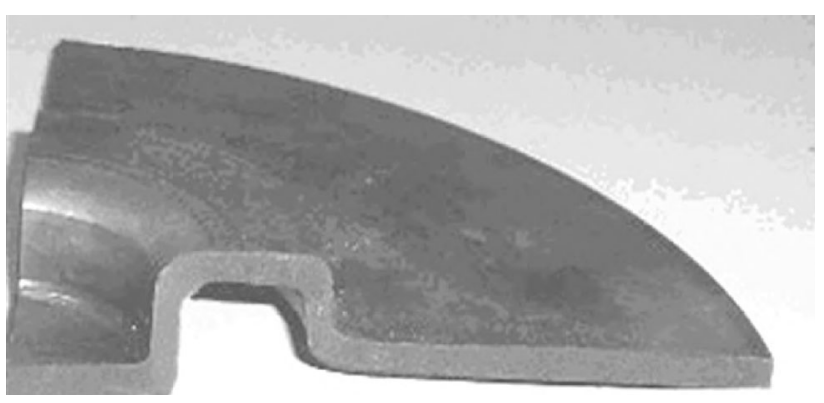

Fig. 25 Press forging hybrid formed parts

$10^{3} / \mathrm{s}$ or even higher, which can greatly improve the forming limit of hard-to-form alloy. Parts formed by PEF possess the merits of little springback, high accuracy, good surface quality and uniform strain distribution. Meanwhile, EMF is flexible and easy to control with simple equipment [76].

Zhao et al. [77] studied electromagnetic microbulging of T2 copper to analyze the effects of discharge voltage, the die cavity depth on the sheet bugling height. Results show that the width and the depth of the microchannels increase with the increase in voltage with a certain die depth; moreover, forming depth reaches the maximum at $7500 \mathrm{~V}$. And then rebound emerges and forming depth decreases. Forming depth and width of channel increase with the decease in die depth at a certain voltage, and forming depth reaches maximum at $0.5 \mathrm{~mm}$ of die depth. Liu and $\mathrm{Yu}$ [7880] conducted a lot of research on the electromagnetic forming of 5052 aluminum alloy. Theoretical analysis and microstructure observation about the electromagnetic dynamic tensile tests demonstrate that the inertia force suppresses the structural instability and improves the material properties by increasing crossing-slip structure. The formed cylindrical parts subjected to electromagneticassisted stamping at different times are shown in Fig. 26.

$\mathrm{Xu}$ et al. [81, 82] investigated free bulging test of AZ31 Mg alloy sheet workpieces with 6111T4 Al foil driver sheet at room temperature with the forming tools shown in Fig. 27. Different bulging height of Mg alloy shown in Fig. 28 can be attained with no drive sheet or drive sheets of various thicknesses under the same capacitance, from which it can be known that the formability of
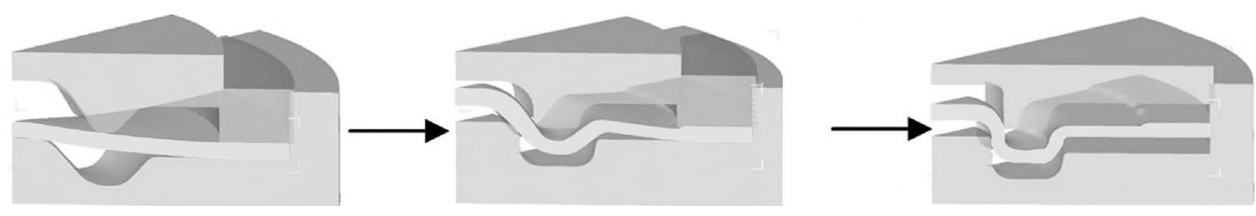

Fig. 24 Technological process of press forging hybrid forming 


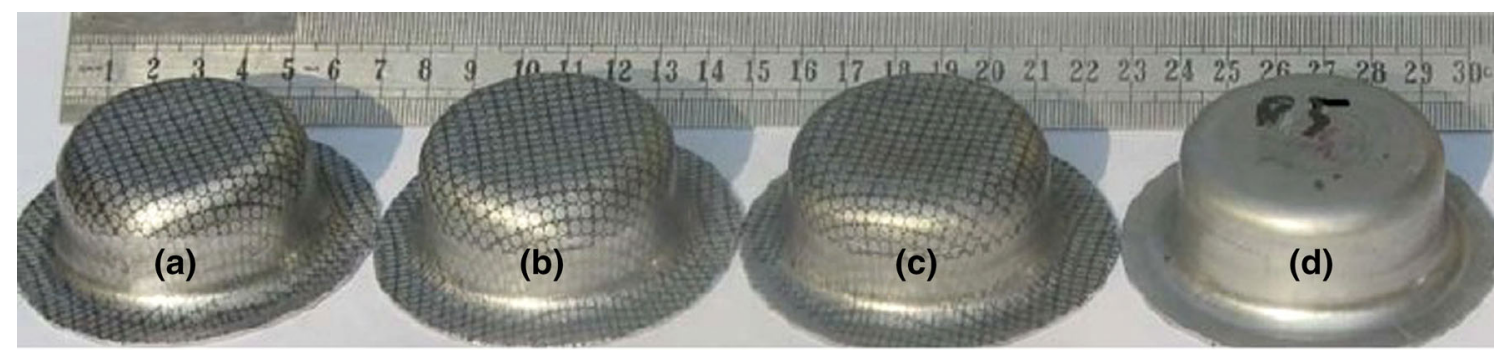

Fig. 26 Cylindrical parts formed with different discharge times: a one time; $\mathbf{b}$ two times; $\mathbf{c}$ three times; $\mathbf{d}$ four times

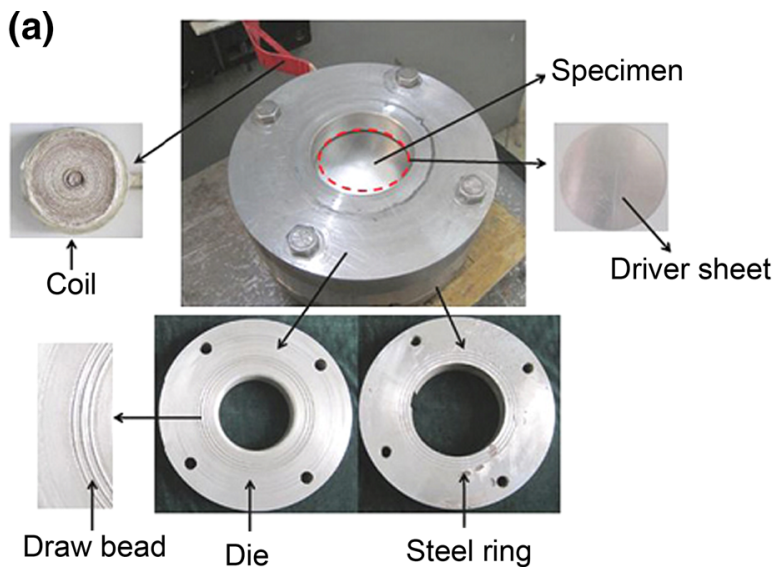

(b)

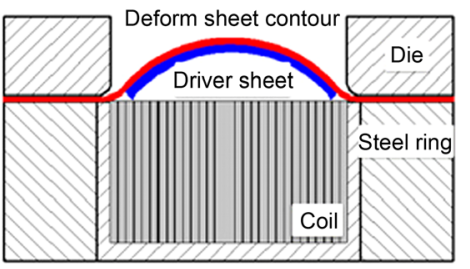

Fig. 27 Tools of electromagnetic forming: a experiment tools; b schematic of diagram

the sheet is best with a thickness $1.0 \mathrm{~mm}$. It also concluded that the inertia force is the main factor to improve forming ability of AZ31 Mg alloy.

The above works are all carried out at the room temperature. However, to form more complex parts, the temperature effect on the EMF should be known clearly. Meng et al. [83-85] clarifie the effect of the temperature and various electrical parameters on the bulging height of AZ31 Mg alloy combining the EMF with warm conditions. If the discharging energy is kept constant, the bulging height decreases first $\left(<150{ }^{\circ} \mathrm{C}\right)$ and then increases $\left(>150{ }^{\circ} \mathrm{C}\right.$ ) from $25{ }^{\circ} \mathrm{C}$ (room temperature) to $230{ }^{\circ} \mathrm{C}$. The higher the temperature is, the better the formability of $\mathrm{Mg}$ alloy will be, but the forming efficiency of WEMF becomes lower. Liu et al. [86] studied electromagnetic blanking of sheet metal parts and analyzed the impact of changes in voltage and electromagnetic coil on blanking fracture modes of foil sheets. To date, the EMF is just in the stage of forming simple parts due to the difficulty of producing uniform and steady EMF on a complex component.

The EMF is a multi-field coupling technology, which has been studied numerically by many researchers. Yu et al. [87, 88] did a lot of numerical simulation of tube electromagnetic forming, which provided a good reference for electromagnetic forming simulation of sheet metal.
Wang and Huang $[89,90]$ conducted numerical simulation about sheet metal electromagnetic forming. Firstly, the model boundary conditions and magnetic pressure distribution were determined through theoretical analysis, and then the deformation of the sheet was analyzed by using ADINA software. Cui et al. [91, 92] established that 2D and 3D finite element model and the sequential coupling method were applied to the free bulging analysis of sheet metal. Mo et al. [93] studied the mechanism of electromagnetic forming concentrator works by the $2 \mathrm{D}$ and $3 \mathrm{D}$ ANSYS finite element model, and analyzed the influence of the structural parameters relative diameter, thickness, radius of the center of the hole on the upper plate concentrator member field force distribution and strength. Li et al. [94] simulated the electromagnetic forming process for the Ti-6Al-4 V sheet with copper driver sheet by loosely coupled numerical simulation method. In the simulation, firstly ANSYS/EMAG was used to calculate on the plate and the magnetic force intensity distribution, and then the magnetic force calculated was introduced into the ABAQUS/Explicit as an initial condition simulation of the plate deformation.

So far, the numerical simulation of electromagnetic forming has made some progresses; however, the numerical simulation model in the past has not reached the level of the fully coupling. The whole coupling means achieving 


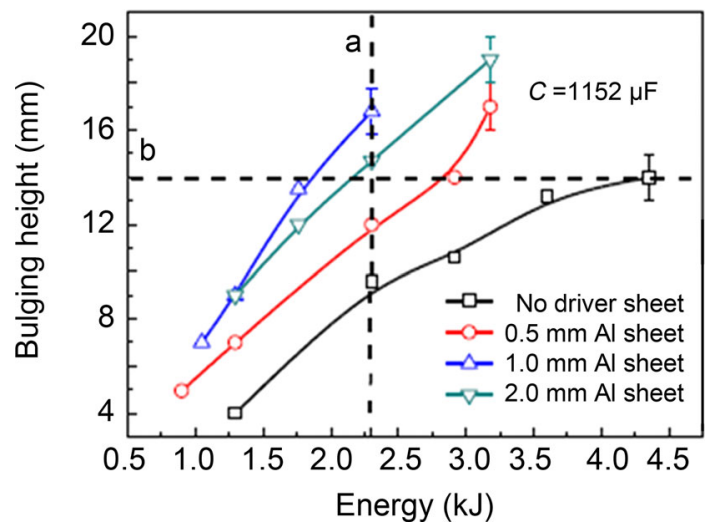

Fig. 28 Effects of driver sheet on the bulging height

the fully coupling of electromagnetics, electrodynamics, plasticity dynamics, thermodynamics and other disciplines. In addition, few researchers consider anisotropy behavior of sheet metal in numerical simulation for electromagnetic forming process, which is the development tendency of the electromagnetic forming technology in the future.

\subsection{Forming Technology of the Special Material}

\subsubsection{Warm Forming Technology of Mg Alloy}

The forming temperature of $\mathrm{Mg}$ alloy sheet metal is generally above $250{ }^{\circ} \mathrm{C}$, called hot forming. This method has the disadvantages such as more energy consuming, equipment and tooling complexity, and scurviness working conditions, which may decline the strength and toughness with respect to grain growth induced by the high temperature. Therefore, it is necessary to make the forming temperature as low as possible. Mori et al. [95, 96] committed to the development of forming technology for $\mathrm{Mg}$ alloy sheet metal and made some progress. However, in order to achieve a desired formability at room temperature, the composition, rolling process and heat treatment of the sheet should be greatly improved, and the increased cost is too high to meet the business requirements [97].

$\mathrm{Mg}$ alloys have a good ductility in the temperature range of $100-230{ }^{\circ} \mathrm{C}$, and the tensile elongation can reach $50 \%$, which is far more larger than the $12 \%$ at room temperature. The essential reason is that a large number of pyramidal slip systems are activated under the warm deformation, and can coordinate lattice strain in the $\mathrm{C}$-axis direction. When the temperature is raised to $230{ }^{\circ} \mathrm{C}$, more pyramidal slip system is activated in the deformation process due to the decrease in critical resolved stress (CRSS), which also lead to a lower strain hardening behavior of material. The flange of part will be thickened during deformation under the compressive stress state, so there is a higher risk of wrinkling if the strain hardening is low. Compared with deformation behavior in the range of 170 and $230{ }^{\circ} \mathrm{C}$, it can be concluded that $170{ }^{\circ} \mathrm{C}$ is sufficient for the sheet metal stamping process. Thus, the deformation temperature should be controlled in the range of $100-170{ }^{\circ} \mathrm{C}$. In this way, it has important applications for simplifying stamping dies, increasing productivity, reducing energy consumption and ensuring stamping quality $[98,99]$.

Zhang et al. $[100,101]$ investigate the non-isothermal deep drawing cylinder of AZ31 Mg alloy and find that nonisothermal drawing LDR of $\mathrm{Mg}$ alloy is not monotonically increasing with sheet temperature rising. As shown in Fig. 29, the LDR of AZ31 reaches the highest value of 2.65 when the forming temperature is $200{ }^{\circ} \mathrm{C}$. When the temperature is increased further, the LDR will decrease. $\mathrm{Mg}$ alloy exhibits good formability with a low punch speed. For example, the LDR of 2.65 can be reached with the drawing speeds of 0.125 and $0.5 \mathrm{~mm} / \mathrm{s}$.

Experimental apparatus shown in Fig. 30 was used to study stamping cylinder of $\mathrm{Mg}$ alloy at different



Fig. 29 LDR curves of non-isothermal deep drawing of AZ31 sheets

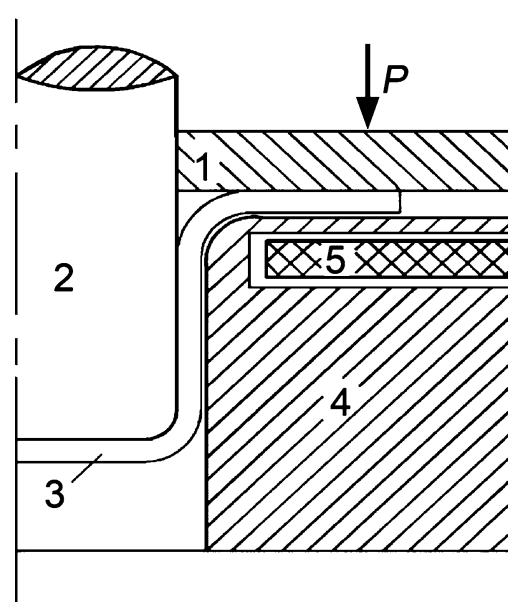

Fig. 30 Schematic of warm deep drawing (1-blank holder; 2punch; 3-blank; 4-die; 5-electric heating elements) 


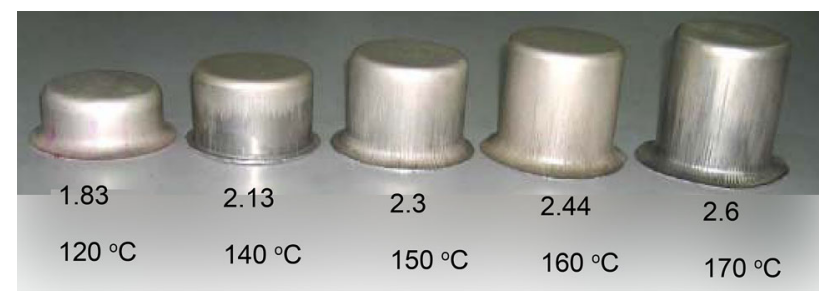

Fig. $31 \mathrm{Mg}$ alloy deep drawing cups and LDR at different temperatures
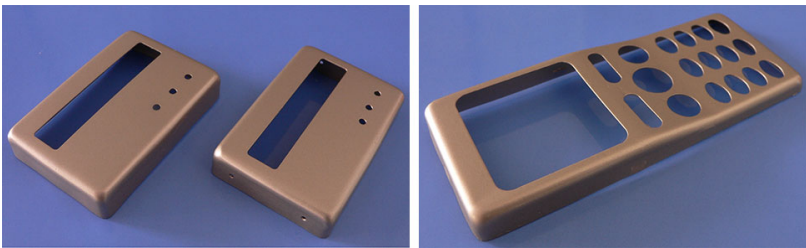

Fig. $32 \mathrm{Mg}$ alloy products of warm forming



Fig. 33 Schematic of warm hydraulic deep drawing (1-blank holder; 2-heater; 3-die; 4-liquid; 5-punch)

temperatures. From the Fig. 31, the LDR can reach 2.60 at the temperature of $170{ }^{\circ} \mathrm{C}$, and it can meet the needs of stamping parts in most conditions. Figure 32 shows some MP3 and mobile phone products produced using the warm forming technology [102-104].

Figure 33 shows warm hydraulic deep drawing device of $\mathrm{Mg}$ alloy. In the experiment, the die is heated to a temperature of $170{ }^{\circ} \mathrm{C}$, and shell-shaped products can be formed with good quality, with reasonable match of punch stroke and hydraulic loading; the productions of mobile phones and digital cameras shell by this technology are shown in Fig. 34 [105].

It is foreseeable that warm forming technology of $\mathrm{Mg}$ alloy has an great industrial meaning, so that $\mathrm{Mg}$ alloy will be widely used in the electronics and automotive fields. In the near future, this technology will quickly be industrial to improve the quality of products greatly.


Fig. 34 Warm hydraulic deep drawing products

\subsubsection{Hot Stamping Technology of Ultra-High-Strength Steel (UHSS)}

In recent years, numerous ultra-high-strength steels (UHSSs) are produced to make the safety components in vehicle, such as the A-pillar, B-pillar and impact beam, to meet the increasing demand for vehicle lightweight, safety and crashworthiness qualities [106]. Hot stamping (HP) is a new forming process, where the sheet is heated to austenitization in a furnace firstly, then pressed and quenched in the closed dies at a high temperature, and finally highstrength parts are formed. Research results indicate that UHSS after heated with a low deformation resistance and good ductility, and the tensile strength of the final component is up to $1500 \mathrm{MPa}$ with small springback compared to cold stamping ones [107].

Ma et al. [108, 109] analyzed hot stamping process of UHSS numerically and experimentally with the independent developed software-King-Mesh Analysis System (KMAS). During the hot stamping process, the phase transformation plastic strain $\varepsilon^{\text {tp }}$ and the phase transformation volume strain $\varepsilon^{\text {tr }}$ were taken into account. Thermomechanical phase transformation coupled constitutive model was adopted [see Eqs. (9-11)] [110-112], and the mechanical properties of the different areas in the hot stamping parts are obtained by using the hybrid rate formula.

$\dot{\varepsilon}=\dot{\varepsilon}(\bar{\sigma}, T)$,

$\dot{\varepsilon}^{\mathrm{tr}}=\dot{\varepsilon}^{\mathrm{tr}}(\bar{\sigma}, T, \varepsilon ; \dot{\bar{\sigma}} \dot{T} \dot{\varepsilon})$,

$\dot{\varepsilon}^{\mathrm{tp}}=\dot{\varepsilon}^{\mathrm{tp}}(\bar{\sigma}, T, \varepsilon ; \dot{\bar{\sigma}} \dot{T} \dot{\varepsilon})$.

Tian et al. [113] studied the influence of temperature and normal pressure on the friction coefficient and the wear in the hot stamping process by a self-designed hightemperature friction test machine, which suggested that the friction coefficient would increase rapidly when the temperature exceeds $600{ }^{\circ} \mathrm{C}$. The influences of die temperature and punch speed on microstructure and the quality of the hot stamping box are investigated by Liu et al. [114]. The microstructure of hot-stamped square-box- 




Fig. 35 Cooling curve of the blank under different contact pressures



Fig. 36 Curve fitting for the equivalent IHTC versus contact pressure

shaped (SBS) component can be fully transformed into martensite phase for the tool temperature of room temperature, while for the tool temperature of $250{ }^{\circ} \mathrm{C}$, such transformation can partially take place for the material of the flange and the sidewall SBS component. Ying et al. [115] studied the effects of die temperature, contact pressure, the air gap and other factors on the hardness gradient distribution in district cooling process of $22 \mathrm{MnB} 5$ parts. Chang et al. [116] studied factors of interfacial heat transfer coefficient (IHTC) during hot stamping of and found that the IHTC of 45 tool steel is about twice of the H13 steels. Figures 35 and 36 show cooling curves and equivalent IHTC under different pressures, respectively. It can be found that with the pressure rising, the IHTC increases rapidly.

The contact pressure and the equivalent IHTC present an exponential relationship, which could be expressed as:

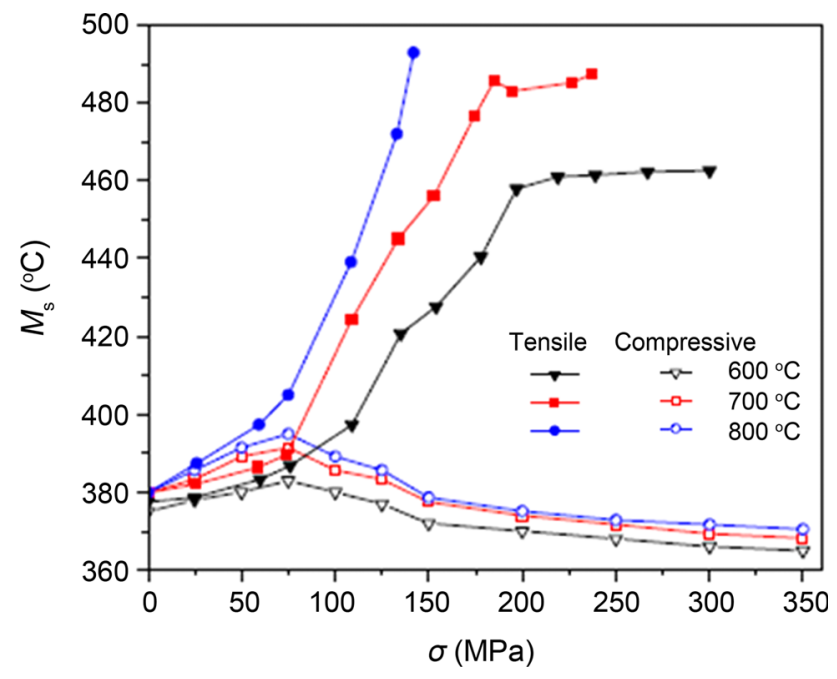

Fig. 37 Effect of tensile/compressive stress $(\sigma)$ and initial forming temperature $(T)$ on the $M_{\mathrm{s}}$ temperature

$\mathrm{IHTC}_{\mathrm{eq}}=1829.6 P^{0.2667}$.

Chang et al. [117] also revealed that the $M_{\mathrm{s}}$ temperature is different under different stress states. As shown in Fig. 37, the $M_{\mathrm{s}}$ can be significantly increased by the tensile stress state. Meanwhile, the $M_{\mathrm{s}}$ point was firstly increased and then decreased under compressive stress. Stress state of different parts position was extracted by simulation, and the Ms can be defined by the stress state,with which the mechanical properties of different parts position were obtained. It is in good agreement with experimental parts. From above research, it is known that the die pressure has a greater impact on IHTC and $M_{\mathrm{s}}$ point. This result was helpful for the understanding of the microstructure and performance of different parts.

Zhang et al. [118, 119] proposed a new technology named as hot stamping - baking toughening forming. The heat of thermal spray process can be made full use of in this technology to achieve carbon partitioning and retain the residual austenite, which improve the elongation of the steel. In order to compensate the decline of the strength caused by carbon partitioning, copper nano-precipitation which does not cause interference with carbon partitioning was used to induce secondary hardening effect in the dislocation martensite phase, and the strength-plastic product could reach $20000 \mathrm{MPa} \%$ with an excellent crash performance. The high-quality lightweight parts can be produced by using this technology, which is shown in Fig. 38. The author provides new ideas and methods for the development of new highstrength steel and hot forming technology of high-strength steels, and it has important practical implications for the integration of part forming and performance. 


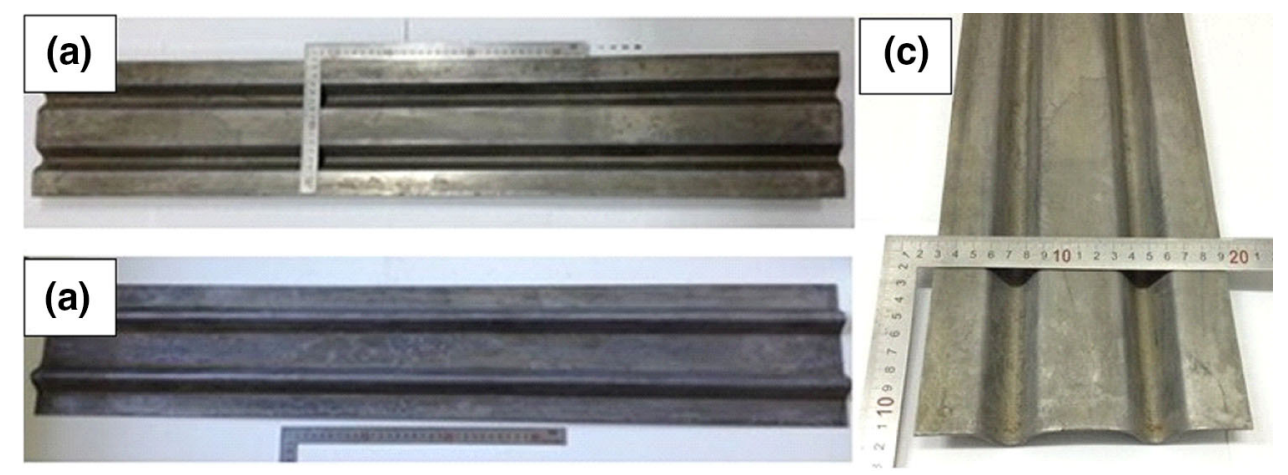

Fig. 38 Stamping parts of new steel

\section{Summary}

This paper shows that many achievements have been reached in China during the last few years. To state the major achievements in the previous sections, the following conclusions are highlighted:

1. Numerical simulation of sheet metal forming: Crystal plasticity theory taking into account twinning and detwinning is established, and the numerical prediction shows good agreement with the experimental measurement. Multi-scale constitutive model considering strain path changes and texture evolution can efficiently capture the real mechanical behavior of HCP material

2. Forming technologies: Solid granular medium forming has been used to produce the bending tubes of which the bending radius is $(R / D)$ equal to 3 and $t / D$ is just 0.01 . The limit drawing ration (LDR) of $\mathrm{Mg}$ alloy can reach 2.60 just at $170{ }^{\circ} \mathrm{C}$ by using the warm forming technology which possess a wide application prospect. A novel UHSS is designed, and a new hot stamping forming technology, hot stamping-baking toughening, is proposed to realize the energy saving and a high ductility of UHSS.

3. In the future, most research works may be focused on macroscopic constitutive model based on physical mechanism; crystal plasticity model considering the graingrain interaction, grain-twinning interaction, twinningtwinning interaction, new forming technologies for special materials, especially for Mg alloy, UHSS, etc.

\section{References}

[1] J. Lévesque, K. Inal, W. Nealek, R.K. Mishar, Int. J. Plast 26, 65 (2010)

[2] B. Clausen, C.N. Tomé, D.W. Brown, S.R. Agnew, Acta Mater. 56, 2456 (2008)
[3] H. Wang, D.P. Wu, C.N. Tomé, Y. Huang, J. Mech. Phys. Solids 58, 594 (2010)

[4] I.J. Polmear, Mater. Sci. Technol. 10, 1 (1994)

[5] H.M. Zhang, X.H. Dong, Q. Wang, Z. Zeng, Comput. Mater. Sci. 54, 208 (2012)

[6] X.L. Geng, K.S. Zhang, Y.Q. Guo, L. Qin, Mater. Sci. Technol. 18, 297 (2010). (in Chinese)

[7] W.Q. Tang, D.Y. Li, Y.H. Peng, Chin. J. Nonferrous Met. 28, 1933 (2014). (in Chinese)

[8] S.B. Yi, C.H. Davies, H.G. Brokmeie, R.E. Bolmaro, K.U. Kainer, J. Homeyer, Acta Mater. 54, 549 (2006)

[9] L. Wu, S.R. Agnew, D.W. Brown, G.M. Stoica et al., Acta Mater. 56, 3699 (2008)

[10] L. Zheng, S.H. Zhang, W.J. He, H.W. Song, D. Helm, Mater. Sci. Technol. 22, 78 (2014). (in Chinese)

[11] L. Zheng, S.H. Zhang, D. Helm, Rear Met. 34, 698 (2015)

[12] L. Zheng, Dissertation, University of Chinese Academy of Sciences, Beijing (2013) (in Chinese)

[13] W. He. Dissertation, University of Chinese Academy of Sciences, Beijing (2013) (in Chinese)

[14] W.J. He, S.H. Zhang, A. Prakash, D. Helm, Comput. Mater. Sci. 82, 464 (2014)

[15] J. Gawad, A. Van Bael, P. Eyckens, G. Samaey, P. Van Houtte, D. Roose, Comput. Mater. Sci. 66, 65 (2013)

[16] X.Y. Yang, L.L. Lang, K.N. Liu, C. Guo, J. Netshape Form. Eng. 7, 22 (2015). (in Chinese)

[17] Y. Ziao, J. Chen, J. Cao, Int. J. Plast 38, 102 (2012)

[18] J. Chen, Y.Z. Xiao, W. Ding, X.H. Zhu, J. Mater. Process. Technol. 215, 151 (2015)

[19] G.S. Ma, M. Wan, X.D. Wu, Chin. J. Nonferrous Met. 44, 246 (2008). (in Chinese)

[20] H. Huang, Y.D. Li, Y.H. Peng, J. Plast. Eng. 17(1), 93 (2010). (in Chinese)

[21] M. Zhong, W.Q. Tang, D.Y. Li, Y.H. Peng, J. Plast. Eng. 18(5), 59 (2011). (in Chinese)

[22] H.W. Swift, J. Mech. Phys. Solids 1, 1 (1952)

[23] R. Hill, J. Mech. Phys. Solids 1, 19 (1952)

[24] S. Stören, J. Rice, J. Mech. Phys. Solids 23, 421 (1975)

[25] X.H. Zhu, K. Weinmann, A.A. Chandra, J. Eng. Mater. Technol. 123, 329 (2001)

[26] Z. Marciniak, K. Kuczynski, Int. J. Mech. Sci. 9, 609 (1967)

[27] P.M. Du, L.L. Lang, B.S. Liu et al., J. Plast. Eng. 18(5), 84 (2011). (in Chinese)

[28] L.L. Lang, G.S. Cai, K.N. Liu, S. Alexandrov, P.M. Du, H. Zheng, Int. J. Mater. Form. 8, 211 (2015)

[29] G. Fang, Q.J. Liu, L.P. Lei, P. Zeng, Trans. Nonferrous Met. Soc. Chin. 22, 343 (2012) 
[30] X.Y. Yang, L.L. Lang, K.N. Liu, G.S. Cai, C. Guo, J. Univ. Aeronaut. Astronaut. 9, 1 (2014). (in Chinese)

[31] H. Takuda, K. Mori, N. Hatta, J. Mater. Process. Technol. 95(1-3), 116 (1999)

[32] J.S. Chen, X.B. Zhou, J. Mech. Eng. 45, 64 (2009). (in Chinese)

[33] C.C. Zhao, Half-Mold Forming Process on Sheet Metal, China patent, ZL200510007167.1, 27 July, 2005 (in Chinese)

[34] M.Y. Cao, G.J. Dong, C.C. Zhao, J. Mech. Eng. 47(14), 62 (2011). (in Chinese)

[35] G.J. Dong, C.C. Zhao, J. Mech. Eng. 48(20), 45 (2012). (in Chinese)

[36] M.Y. Cao, C.C. Zhao, G.J. Dong, Chin. J. Nonferrous Met. 22, 2992 (2012). (in Chinese)

[37] M.Y. Cao, C.C. Zhao, G.J. Dong, H.B. Hao, J. Mech. Eng. 49(2), 42 (2013). (in Chinese)

[38] L.H. Guo, S.J. Yang, H. Jiao, Thin Walled Struct. 73, 281 (2013)

[39] H. Lia, H. Yang, Z.Y. Zhang, G.J. Li, N. Liu, T. Welo, J. Mater. Process. Technol. 214, 445 (2014)

[40] S.H. Zhang, Z.R. Wang, Y. Xu, Z.T. Wang, L.X. Zhou, J. Mater. Process. Technol. 151, 237 (2004)

[41] Z.J. Wang, B.X. Yuan, Trans. Nonferrous Met. Soc. China 24, 462 (2014)

[42] Z.J. Wang, D.L. Cheng, Trans. Nonferrous Met. Soc. China 18, 1189 (2008)

[43] Y.M. Zhou, J.X. Lei, Hot Working Technol. 42, 87 (2013). (in Chinese)

[44] E. Chu, Y. Xu, Int. J. Mech. Sci. 43, 1421 (2001)

[45] B.G. Chen, S.J. Yuan, Y.C. Xu, Z.R. Wang, J. Plast. Eng. 17(6), 40 (2010). (in Chinese)

[46] L.H. Lang, Y.M. Wang, Y.S. Xie, H.L. Li, X.P. Hui, J. Plast. Eng. 18(5), 33 (2011). (in Chinese)

[47] B.G. Chen, B. Song, Z.B. Huang, X.T. He, Y.C. Xu, S.J. Yuan, Forg. Stamp. Technol. 40(1), 43 (2015). (in Chinese)

[48] L.H. Lang, N. Xu, Y.M. Wang, C. Yuan, Y.Q. Xu, Z.H. Yang, T.W. Shao, X.M. Duan, Forg. Stamp. Technol. 38(2), 21 (2013). (in Chinese)

[49] B.G. Chen, Y.C. Xu, Mater. Sci. Technol. 19, 17 (2011). (in Chinese)

[50] J.F. Lin, F. Li, J.F. Zhang, Trans. Nonferrous Met. Soc. China 22, s232 (2012)

[51] Z.J. Wang, H. Song, Z. Wang, Trans. Nonferrous Met. Soc. China 18, 72 (2008)

[52] Z.J. Wang, T.J. Gao, Trans. Nonferrous Met. Soc. China 18, 285 (2008)

[53] H.T. Wang, L. Gang, X.H. Shen, Y. Xu, J. Mech. Eng. 46(12), 76 (2010). (in Chinese)

[54] X.C. Mao, J. Plast. Eng. 19(4), 43 (2012). (in Chinese)

[55] H.T. Wang, L. Gang, X.H. Shen, L.G. Li, Acta Aeronaut. Astronaut. Sin. 31, 1266 (2010). (in Chinese)

[56] X.J. Liu, Y.L. Cong, F. Li, Y.C. Xu, S.J. Yuan, J. Cent. South. Univ. (Sci. Technol.) 41, 917 (2010). (in Chinese)

[57] H.T. Wang, J. Zhang, L. Gao, X.H. Shen, J. Mech. Eng. 47(16), 73 (2011). (in Chinese)

[58] H.T. Wang, G. Lin, X.H. Shen, M.H. Chen, Chin. J. Nonferrous Met. 20, 840 (2010). (in Chinese)

[59] G.J. Dong, C.C. Zhao, Y.X. Peng, Y. Li, Chin. J. Mech. Eng. 28, 580 (2015). (in Chinese)

[60] T. Jin, J.X. Lei, S.H. Qiu, J. Plast. Eng. 17(4), 53 (2010). (in Chinese)

[61] S.D. Zhao, J.H. Yuan, J. Wang, China Met. Form. Equip. Manuf. Technol. 44, 44 (2006). (in Chinese)

[62] Y. Zhu, M. Wan, Y.K. Zhou, Acta Aeronaut. Astronaut. Sin. 32, 552 (2011). (in Chinese)
[63] B. Meng, M. Wan, X.D. Wu, S. Yuan, X.D. Xu, J. Liu, Int. J. Mech. Sci. 79, 143 (2014)

[64] A.Y. Tai, H.T. Wang, J. Yan, J. Anhui Univ. Technol. (Nat. Sci.) 31, 353 (2014). (in Chinese)

[65] D.K. Sun, S.Q. Wang, H.J. Yu, F. Li, Q.W. Qu, Mech. Sci. Technol. Aero. Eng. 33, 541 (2014). (in Chinese)

[66] S.X. Ding, L.H. Lang, L. Huang, J. Netshape Form. Eng. 6(3), 31 (2014). (in Chinese)

[67] X.J. Liu, W.Y. Liu, paper present in the 6th International Forum Strategic Technology (Haerbin, Aug. 22-24, 2011), p. 181

[68] Y.Y. Liu, Y. Xu, H. Wang, J. Li, J. Nanjing Univ. Aeronaut. Astronautics. 44, 101 (2012). (in Chinese)

[69] X.J. Liu, J.R. Fu, Q.R. Pan, P.H. Liu, paper present in the 2013 International Conference on Material Science and Environmental Engineering (MSEE) (Wuhan, 2013), p. 514

[70] S.H. Zhang, L.X. Zhou, Z.T. Wang, J. Plast. Eng. 15(2), 31 (2008). (in Chinese)

[71] S.H. Zhang, C. Sun, Z.T. Wang, Trans. Nonferrous Met. Soc. China 18, 269 (2008)

[72] X.Y. Wang, J.C. Xia, Z.M. Chen, J. Plast. Eng. 15(4), 180 (2008). (in Chinese)

[73] X.Y. Wang, W.T. Luo, J.C. Xia, Forg. Technol. 24(5), 43 (2009). (in Chinese)

[74] X.Y. Wang, Y.K. Ou, J.C. Xia, Forg. Technol. 34(4), 73 (2009). (in Chinese)

[75] Y.Y. Peng, Z.T.Z. Gong, S.W. Yang, P. Li, K.M. Xue, J. Plast. Eng. 22(1), 1 (2015). (in Chinese)

[76] X.W. Chen, W.P. Wang, M. Wan, W.R. Xiong, Rare Met. Mater. Eng. 44, 514 (2015). (in Chinese)

[77] Q.J. Zhao, C.J. Wang, H.P. Yu, G. Bin, D.B. Shan, C.F. Trans, Nonferrous Met. Soc. Chin. 21, 461 (2011)

[78] D.H. Liu, H.P. Yu, C.F. Li, J. Plast. Eng. 20(3), 70 (2013). (in Chinese)

[79] D.H. Liu, H.P. Yu, C.F. Li, Mater. Sci. Eng., A 551, 280 (2012)

[80] D.H. Liu, H.P. Yu, C.F. Li, Acta Metall. Sin. 48, 519 (2012). (in Chinese)

[81] J.R. Xu, H.P. Yu, J.J. Cui, C.F. Li, Mater. Sci. Eng., A 569, $150(2013)$

[82] J.R. Xu, H.P. Yu, C.F. Li, J. Mater. Eng. Perform. 22, 1179 (2013)

[83] Z.H. Meng, S.Y. Huang, J.H. Hu, Z.L. Xia, X.X. Feng, J. Mech. Eng. 47(10), 38 (2011). (in Chinese)

[84] Z.H. Meng, S.Y. Huang, J.H. Hu, W. Huang, Z.L. Xia, J. Mater. Process. Technol. 211, 863 (2011)

[85] F. Feng, S.Y. Huang, J.H. Hu, Z.H. Meng, J. Plast. Eng. 20(6), 56 (2013). (in Chinese)

[86] Q.S. Liu, S.H. Huang, Z.H. Chang, Forg. Stamp. Technol. 27(1), 36 (2002). (in Chinese)

[87] H.P. Yu, C.F. Li, Z.H. Zhao, J. Mater. Process. Technol. 209, 1053 (2009)

[88] H.P. Yu, C.F. Li, J.H. Deng, J. Mater. Process. Technol. 209, 707 (2009)

[89] L.F. Wang, S.H. Huang, J. Syst. Simul. 18, 757 (2006). (in Chinese)

[90] L.F. Wang, Z.Y. Chen, C.X. Li, S.Y. Huang, Int. J. Adv. Manuf. Technol. 30, 395 (2006)

[91] X.H. Cui, J.H. Mo, B. Wang, H.W. Zhi, J. Mech. Eng. 47(16), 45 (2011). (in Chinese)

[92] X.H. Cui, J.H. Mo, Z. Ying, Trans. Nonferrous Met. Soc. China 22, 164 (2012)

[93] J.H. Mo, B. Wang, X.H. Cui, W.Z. He, J. Plast. Eng. 18(1), 36 (2011). (in Chinese)

[94] F.Q. Li, J.H. Mo, J.J. Li, L. Huang, H.Y. Zhou, Mater. Des. 64, 1575 (2013) 
[95] K. Iwanaga, H. Tashiro, H. Okamoto, K. Shimizu, J. Mater. Process. Technol. 155-156, 1313 (2004)

[96] K. Mori, T. Tsuji, Ann. CIRP 56(1), 285 (2007)

[97] S.H. Zhang, Z.T. Wang, L.X. Zhou, Y.C. Xu, Mater. Rev. 20(8), 114 (2006). (in Chinese)

[98] G.S. Song, S.H. Zhang, L. Zheng, L. Ruan, J. Alloys Compd. 509, 6481 (2011)

[99] S.H. Zhang, L.M. Ren, L.X. Zhou, Y.C. Xu, G. Palumbo, L. Tricarico, Mater. Sci. Forum 546-549, 333 (2007)

[100] S.H. Zhang, K. Zhang, Y.C. Xu, Z.T. Wang, Y. Xu, Z.G. Wang, J. Mater. Process. Technol 185, 147 (2007)

[101] L.M. Ren, S.H. Zhang, G. Palumbo, D. Sorgente, L. Tricarico, Mater. Sci. Eng., A 499, 40 (2009)

[102] S.H. Zhang, G.S. Song, H. Song, M. Cheng, J. Mech. Eng. 48(18), 28 (2012)

[103] K.F. Zhang, D.L. Yin, D.Z. Wu, S.X. Jiang, Chin. J. Nonferrous Met. 12, 1505 (2003)

[104] D.L. Yin, K.F. Zhang, D.Z. Wu, Mater. Sci. Technol. 12, 87 (2004)

[105] K.F. Zhang, D.L. Yin, D.Z. Wu, Int. J. Mach. Tools Manuf 46, 1276 (2006)

[106] H. Karbasian, A.E. Tekkaya, J. Mater. Process. Technol. 210, $2103(2010)$

[107] Y. Shan, Forg. Technol. 40(2), 22 (2015). (in Chinese)
[108] N. Ma, P. Hu, K.K. Yan, W. Guo, X.B. Meng, X.J. Zhai, J. Mech. Eng. 46(14), 69 (2010). (in Chinese)

[109] P. Hu, N. Ma, Adv. Mech. 4, 310 (2011). (in Chinese)

[110] G.W. Greenwood, R.H. John, Proc. R. Soc. London 283, 403 (1965)

[111] S. Dens, E. Gautiter, A. Simon, G. Beck, Mater. Sci. Technol. 1, 805 (1985)

[112] T. Kakeshita, C.M. Wayman, Mater. Sci. Eng., A 147, 85 (1991)

[113] X.W. Tian, Y.S. Zhang, Z. Wang, H.Q. Li, L. Wang, B. Zhu, J. Li, Adv. Mater. Res. 421, 147 (2012)

[114] W. Liu, H. Liu, Z. Xing, G. Liu, J. Bao, Trans. Nonferrous Met. Soc. China 22, 534 (2012)

[115] Y. Liang, Dissertation, Dalian University of Technology (2013) (in Chinese)

[116] Y. Chang, X. Tang, K. Zhao, P. Hu, Y. Wu, J. Mater. Process. Technol. 10, 1 (2014)

[117] Y. Chang, X.D. Li, K.M. Zhao, C.Y. Wang, G.J. Zheng, P. Hu, H. Dong, Mater. Sci. Eng., A 629, 1 (2015)

[118] T. Lin, H.W. Song, S.H. Zhang, M. Cheng, W.J. Liu, Chin. J. Mater. Res. 28, 88 (2014). (in Chinese)

[119] M. Cheng, H.W. Song, X. Li, S.H. Zhang, M. Cheng, T. Lin, Mater. Sci. Forum 788, 340 (2014) 